\title{
Clinical and molecular diagnosis, screening and management of Beckwith-Wiedemann syndrome: an international consensus statement
}

Frédéric Brioude', Jennifer M. Kalish², Alessandro Mussa ${ }^{3,4}$, Alison C. Foster ${ }^{5,6}$, Jet Bliek, Giovanni Battista Ferrero3, Susanne E. Boonen ${ }^{8}$, Trevor Cole ${ }^{5}$, Robert Baker ${ }^{9}$, Monica Bertoletti' ${ }^{10}$, Guido Cocchi ${ }^{11}$, Carole Coze ${ }^{12}$, Maurizio De Pellegrin ${ }^{13}$, Khalid Hussain ${ }^{14}$, Abdulla Ibrahim ${ }^{15}$, Mark D. Kilby ${ }^{16,17}$, Malgorzata Krajewska-Walasek ${ }^{18}$, Christian P. Kratz ${ }^{19}$, Edmund J. Ladusans ${ }^{20}$, Pablo Lapunzina ${ }^{21,22}$, Yves Le Bouc ${ }^{1}$, Saskia M. Maas ${ }^{7}$, Fiona Macdonald ${ }^{23}$, Katrin Õunap ${ }^{24}$, Licia Peruzzi25,26, Sylvie Rossignol27, Silvia Russo ${ }^{28}$, Caroleen Shipster ${ }^{29}$, Agata Skórka ${ }^{18,30}$, Katrina Tatton-Brown ${ }^{31}$, Jair Tenorio ${ }^{21,22}$, Chiara Tortora ${ }^{32}$, Karen Grønskov ${ }^{33}$, Irène Netchine ${ }^{1}$, Raoul C. Hennekam ${ }^{34}$, Dirk Prawitt ${ }^{35}$, Zeynep Tümer ${ }^{33}$, Thomas Eggermann ${ }^{36}$, Deborah J. G. Mackay ${ }^{37}$, Andrea Riccio ${ }^{38}$ and Eamonn R. Maher ${ }^{39}$

Abstract | Beckwith-Wiedemann syndrome (BWS), a human genomic imprinting disorder, is characterized by phenotypic variability that might include overgrowth, macroglossia, abdominal wall defects, neonatal hypoglycaemia, lateralized overgrowth and predisposition to embryonal tumours. Delineation of the molecular defects within the imprinted $11 \mathrm{p} 15.5$ region can predict familial recurrence risks and the risk (and type) of embryonal tumour. Despite recent advances in knowledge, there is marked heterogeneity in clinical diagnostic criteria and care. As detailed in this Consensus Statement, an international consensus group agreed upon 72 recommendations for the clinical and molecular diagnosis and management of BWS, including comprehensive protocols for the molecular investigation, care and treatment of patients from the prenatal period to adulthood. The consensus recommendations apply to patients with Beckwith-Wiedemann spectrum (BWSp), covering classical BWS without a molecular diagnosis and BWS-related phenotypes with an 11p15.5 molecular anomaly. Although the consensus group recommends a tumour surveillance programme targeted by molecular subgroups, surveillance might differ according to the local health-care system (for example, in the United States), and the results of targeted and universal surveillance should be evaluated prospectively. International collaboration, including a prospective audit of the results of implementing these consensus recommendations, is required to expand the evidence base for the design of optimum care pathways.

Correspondence to E.R.M. erm1000@medschl.cam. ac.uk

doi: $10.1038 /$ nrendo. 2017.166 Published online 29 Jan 2018
Beckwith-Wiedemann syndrome (BWS) is a multisystem human genomic imprinting disorder with variable clinical expression and complex molecular aetiology ${ }^{1}$. BWS is an overgrowth syndrome, with patients often presenting with macroglossia, abdominal wall defects, hemihyperplasia (lateralized overgrowth), enlarged abdominal organs and an increased risk of embryonal tumours during early childhood. BWS is caused mainly by genetic or epigenetic defects within the chromosome $11 \mathrm{p} 15.5$ region $^{2}$. This region contains imprinted genes, such as CDKN1C and IGF2, which are strong regulators of fetal growth. Although BWS might present prenatally or in adult life, it is most commonly diagnosed in the neonatal period or in early childhood, with an estimated prevalence of 1 affected child 
per 10,340 live births ${ }^{3}$. Since the first descriptions half a century ago, a PubMed search (conducted on 15 November 2016) for "Beckwith Wiedemann", "Wiedemann Beckwith" or "EMG syndrome" yielded >1,500 BWS-related articles. However, clinical practice varies regarding the diagnosis and care of individuals with BWS.

To address these issues, the European Cooperation in Science and Technology (COST)-funded European Network for Congenital Imprinting Disorders initiated a BWS consensus programme that involved an extensive literature review, preparation and critical appraisal of draft documents and a final face-to-face consensus meeting involving invited experts and patient group representatives. This effort produced a series of consensus recommendations for the diagnosis and care of individuals with the newly defined Beckwith-Wiedemann spectrum (BWSp), which are presented in this Consensus Statement.

\section{Author addresses}

${ }^{1}$ Sorbonne Université, Pierre and Marie Curie-Paris VI University (UPMC) Université Paris 06, INSERM UMR_S938 Centre de Recherche Saint-Antoine (CRSA), APHP Hôpital Trousseau, Explorations Fonctionnelles Endocriniennes, 26 Avenue du Docteur Arnold Netter, F-75012 Paris, France.

${ }^{2}$ Division of Human Genetics, Children's Hospital of Philadelphia and the Department of Pediatrics at the Perelman School of Medicine, University of Pennsylvania, Philadelphia, PA 19104, USA.

${ }^{3}$ Department of Public Health and Pediatric Sciences, University of Torino, Piazza Polonia 94, 10126 Torino, Italy.

${ }^{4}$ Neonatal Intensive Care Unit, Department of Gynaecology and Obstetrics, Sant'Anna Hospital, Città della Salute e della Scienza di Torino, Corso Spezia 60, 10126 Torino, Italy. ${ }^{5}$ Birmingham Health Partners, West Midlands Regional Genetics Service, Birmingham Women's and Children's National Health Service (NHS) Foundation Trust, Birmingham B15 2TG, UK.

${ }^{6}$ Institute of Cancer and Genomic Sciences, College of Medical and Dental Sciences, University of Birmingham, Birmingham B15 2TT, UK.

${ }^{7}$ Department of Clinical Genetics, Academic Medical Center, University of Amsterdam, PO Box 70571007 MB Amsterdam,

The Netherlands.

${ }^{8}$ Clinical Genetic Unit, Department of Pediatrics, Zealand University Hospital, Sygehusvej 104000 Roskilde, Denmark. ${ }^{9}$ Beckwith-Wiedemann Support Group UK, The Drum and Monkey, Wonston, Hazelbury Bryan, Sturminster Newton, Dorset DT10 2EE, UK. ${ }^{10}$ Italian Association of Beckwith-Wiedemann syndrome (AIBWS) Piazza Turati, 3, 21029 , Vergiate (VA), Italy.

${ }^{11}$ Alma Mater Studiorum, Bologna University, Paediatric Department, Neonatology Unit, Via Massarenti 11, 40138 Bologna BO, Italy. ${ }^{12}$ Aix-Marseille Univ et Assistance Publique Hôpitaux de Marseille (APHM), Hôpital d'Enfants de La Timone, Service d'Hématologie-Oncologie Pédiatrique, 264 Rue Saint Pierre, 13385 Marseille, France. ${ }^{13}$ Pediatric Orthopaedic Unit IRCCS Ospedale San Raffaele, Milan, Via Olgettina Milano, 60, 20132 Milano MI, Italy.
${ }^{14}$ Department of Paediatric Medicine, Division of Endocrinology, Sidra Medical and Research Center, Al Gharrafa Street, Ar-Rayyan, Doha, Qatar.

${ }^{15}$ Department of Plastic and Reconstructive Surgery, North Bristol National Health Service (NHS) Trust, Southmead Hospital, Bristol BS10 5NB, UK.

${ }^{16}$ Institute of Metabolism and Systems Research, College of Medical and Dental Sciences, University of Birmingham, Birmingham, B15 2TT, UK.

${ }^{17}$ Fetal Medicine Centre, Birmingham Women's and Children's National Health Service (NHS) Foundation Trust, Edgbaston, Birmingham, B15 2TG, UK.

${ }^{18}$ Department of Medical Genetics, The Children's Memorial Health Institute,

20, 04-730, Warsaw, Poland.

${ }^{19}$ Pediatric Hematology and Oncology,

Hannover Medical School, Carl-Neuberg-

Strasse 1 30625, Hannover, Germany.

${ }^{20}$ Department of Paediatric Cardiology, Royal Manchester Children's Hospital, Manchester, M13 8WL UK.

${ }^{21}$ Instituto de Genética Médica y Molecular (INGEMM)-IdiPAZ, Hospital Universitario La Paz-UAM Paseo de La Castellana, 261, 28046, Madrid, Spain.

${ }^{22}$ CIBERER, Centro de Investigación Biomédica en Red de Enfermedades Raras, ISCIII, Calle de Melchor Fernández Almagro, 3, 28029, Madrid, Spain.

${ }^{23}$ West Midlands Regional Genetics Laboratory, Birmingham Women's and Children's National Health Service (NHS) Foundation Trust,

Birmingham, B15 2TG UK.

${ }^{24}$ Department of Clinical Genetics, United Laboratories, Tartu University Hospital and Department of Clinical Genetics, Institute of Clinical Medicine, University of Tartu,

L. Puusepa 2, 51014, Tartu, Estonia.

${ }^{25}$ European Society for Paediatric Nephrology (ESPN), Inherited Kidney Disorders Working Group.

${ }^{26} \mathrm{AOU}$ Città della Salute e della Scienza di Torino, Regina Margherita Children's Hospital, Turin, Italy.

${ }^{27}$ Service de Pédiatrie, Hôpitaux Universitaires de Strasbourg, Laboratoire de Génétique Médicale, INSERM U1112 Avenue Molière 67098 STRASBOURG Cedex, Fédération de
Médecine Translationnelle de Strasbourg (FMTS), Université de Strasbourg, 4 Rue Kirschleger, 67000 Strasbourg, France. ${ }^{28}$ Medical Cytogenetics and Molecular Genetics Laboratory, Centro di Ricerche e Tecnologie Biomediche IRCCS, Istituto Auxologico Italiano, Via Zucchi 18, 20095 Cusano, Milan, Italy. ${ }^{29} \mathrm{Great}$ Ormond Street Hospital for Children National Health Service (NHS) Foundation Trust, London, WC1N 3JH, UK.

${ }^{30}$ Department of Pediatrics, The Medical University of Warsaw, Zwirki i Wigury 63a, 02-091 Warszawa, Poland.

${ }^{31}$ South West Thames Regional Genetics Service and St George's University of London and Institute of Cancer Research, London, SW17 0RE, UK.

${ }^{32}$ Regional Center for CLP, Smile House, San Paolo University Hospital, Via Antonio di Rudini, 8, 20142, Milan, Italy.

${ }^{33}$ Kennedy Center, Department of Clinical Genetics, Copenhagen University Hospital, Rigshospitalet, Blegdamsvej 9, 2100

Copenhagen, Denmark.

${ }^{34}$ Department of Pediatrics, Emma Children's Hospital, Academic Medical Center, University of Amsterdam, Meibergdreef 9, 1105 AZ Amsterdam-Zuidoost, Amsterdam, The Netherlands.

${ }^{35}$ Center for Pediatrics and Adolescent Medicine, Johannes Gutenberg University Medical Center, Langenbeckstr. 1, D-55101, Mainz, Germany.

${ }^{36}$ Institute of Human Genetics, University Hospital, Technical University of Aachen, Templergraben 55, 52062, Aachen, Germany. ${ }^{37}$ Human Development and Health, Faculty of Medicine, University of Southampton, Southampton SO17 1BJ, UK.

${ }^{38}$ Department of Environmental, Biological, and Pharmaceutical Sciences and Technologies(DiSTABiF), University of Campania Luigi Vanvitelli, Caserta and Institute of Genetics and Biophysics

“A. Buzzati-Traverso" - CNR, Via Pietro Castellino, 111,80131, Naples, Italy.

${ }^{39}$ Department of Medical Genetics, University of Cambridge and National Institute for Health Research (NIHR) Cambridge Biomedical Research Centre and Cancer Research UK Cambridge Centre, Cambridge Biomedical Campus, Cambridge, CB2 0QQ, UK. 


\section{Methods}

A PubMed search using the keywords "Beckwith Wiedemann", "Wiedemann Beckwith" or "EMG syndrome" yielded articles of interest that were selected on the basis of the abstracts, with particular consideration given to the number of patients included and the description of the molecular mechanisms. Only articles mentioning the molecular mechanisms were retained. Articles were then reviewed by at least two experts and sorted into three groups: clinical diagnosis (group 1); molecular diagnosis (group 2); and clinical management (group 3).

The international BWS consensus group comprised 41 participants from 36 institutions across 11 countries, predominantly based in Europe, including clinicians, clinical and research scientists and patient group representatives with expertise in different aspects of BWS (clinical and molecular geneticists, paediatric endocrinologists, oncologists, orthopaedists, oro-facial surgeons and nephrologists). A modified Delphi consensus process was adopted ${ }^{4}$. Discussions occurred via conference calls, email communications and file exchanges. Two face-to-face meetings were held: a preliminary meeting of 11 participants (including one patient group representative) in February 2016 to identify the key issues to be addressed by the consensus group and a plenary 3-day meeting involving 35 participants (including 2 patient group representatives) in March 2017. During this plenary meeting, experts participated in one of the three subgroups (clinical, molecular or management) on the basis of their field of expertise, discussed the draft consensus documents and formulated and voted on the consensus recommendations (BOX 1). This Consensus Statement summarizes the outcome of these discussions and is divided into three subject areas: clinical aspects; molecular aspects; and care and management.

\section{Clinical aspects of BWS}

Since the seminal descriptions by Beckwith ${ }^{5}$ and Wiedemann ${ }^{6}$ in the 1960 s, there have been many attempts to define BWS by use of various clinical criteria that have been reported with different prevalences in cohorts of BWS (Supplementary information S1 (table)); however, no agreed clinical definition of BWS has emerged. Since the findings in the 1990s of molecular abnormalities of chromosome 11p15.5 in BWS ${ }^{7-9}$,

\section{Box $1 \mid$ Details of the consensus voting process}

For voting on individual recommendations, participants $(n=33)$ selected from the following options (patient group representatives did not vote):

- A. Evidence or general agreement allow full agreement with the recommendation

- B. Evidence or general agreement are in favour of the recommendation

- C. Evidence or general agreement are weak for the recommendation

- D. There is not enough evidence or general agreement to agree with the recommendation

Depending on the proportion of votes received, the strength of the recommendation was recorded as follows:

,$-+ 26-49 \%$ of the votes

,$-++ 50-69 \%$ of the votes

,$-+++ \geq 70 \%$ of the votes it has been recognized that these genetic and epigenetic changes are frequently mosaic and lead to a range of clinical phenotypes. These include 'classical BWS' (OMIM \#130650), which is characterized by macroglossia, anterior abdominal wall defects and prenatal and postnatal overgrowth, among other symptoms (Supplementary information S1 (table)), and some cases of isolated lateralized overgrowth (previously called 'isolated hemihypertrophy' or 'isolated hemihyperplasia'; OMIM $\# 235000)^{10}$ in addition to patients with a chromosome 11 p15.5 molecular anomaly who do not fit into these first two groups, a condition termed 'atypical BWS' by this consensus group. Given the overlapping phenotypes and common molecular mechanisms between these groups, the consensus group decided that these phenotype and/or genotype combinations could be best classified as parts of the BWSp (TABLE 1, R1)and that the recommendations of this consensus should be applied to individuals with BWSp (FIG. 1; TABLE 1).

\section{Clinical features of BWSp}

BWS has classically been characterized by macroglossia, macrosomia, abdominal wall defects and an increased risk of embryonal tumours ${ }^{11-17}$. There is growing recognition that not all patients with BWS display all of these phenotypic features and that patients have remained undiagnosed because they did not present with one of these features, such as macrosomia, which was initially considered as a cardinal feature but is present in only onehalf of the patients with an 11p15.5 molecular defect ${ }^{18,19}$. The clinical features outlined as part of the consensus BWSp scoring system include features that, when present, are more likely to lead to a positive diagnosis (termed 'cardinal features'), including macroglossia, exomphalos, lateralized overgrowth, multifocal Wilms tumour or nephroblastomatosis, hyperinsulinism and specific pathology findings (such as adrenal cytomegaly or placental mesenchymal dysplasia) (BOX 2). Macrosomia has been defined according to different criteria in different clinical cohorts ${ }^{11-13,15-17}$, making it challenging to assess the role it has as a cardinal feature. Lateralized overgrowth is the novel term for hemihypertrophy (or hemihyperplasia), which is defined as asymmetric overgrowth of part of the body ${ }^{10}$. Embryonal tumours such as Wilms tumours and hepatoblastoma can occur outside of the diagnostic scope of BWSp; however, multifocal Wilms tumours are more likely to occur in BWSp. As a cardinal feature, hyperinsulinism is defined as prolonged hypoglycaemia in the context of elevated insulin levels that last $>1$ week and/or require escalated treatment ${ }^{20}$, while transient hypoglycaemia resolves without the need for further intervention. Although pathology findings cannot always be evaluated (especially when BWSp is not suspected prenatally or at birth and when placental samples are not collected), the diagnosis of BWSp should be considered in cases of adrenal cortex cytomegaly, placental mesenchymal dysplasia and pancreatic adenomatosis ${ }^{5}$. Additionally, if samples are available (especially from the placenta after birth) and the diagnosis is being considered, pathological investigation can be beneficial in making the clinical diagnosis. 
Table 1 | Consensus recommendations of the clinical working group

\section{BWSp scoring system and clinical indications for molecular testing for BWS}

1 BWSp is usually caused by dysregulation of the chromosome $11 \mathrm{p} 15$ imprinted region and involves overgrowth in multiple tissues, often in a mosaic state. BWSp encompasses a range of phenotypes, and children might present with one or more features (summarized in BOX 2). Classical BWS and lateralized overgrowth ('hemihypertrophy and/or hemihyperplasia') are considered subsets of the BWSp (FIG. 1). A third subset is defined as patients with an 11p15 anomaly who do not fit into these first two groups

2 There have been many proposed systems to define classical BWS, which have suggested combinations of macroglossia, omphalocele/exomphalos and/or (asymmetric) overgrowth. Although often associated, increased height and/or weight (macrosomia) are no longer considered a cardinal feature of BWS. For simplicity and consistency, we have developed consensus criteria (TABLE 2): a score of $\geq 4$ must be reached for a diagnosis of classical BWS. Children who meet these criteria would be considered to have BWSp, even if an 11p15 anomaly is not identified

3 BWSp lateralized overgrowth is defined as a marked increase in the length and/or girth of most or all of one side of the body compared with its contralateral side in addition to an $11 \mathrm{p} 15$ abnormality. A child with an 11p15 anomaly who does not meet the criteria for classical BWS or BWSp lateralized overgrowth is still considered to be part of the BWSp. There are currently insufficient data to determine the management guidelines for patients with lateralized overgrowth without an identified 11p15 anomaly in tested samples; this group falls outside the scope of this consensus

4 We recommend molecular testing in anyone suspected of being in the BWSp (lateralized overgrowth, classical BWS or features in TABLE 2). For simplicity, we recommend molecular testing for any patient with $\geq 2$ points. In cases where isolated omphalocele and/or exomphalos is present, testing can be considered at the discretion of the physician. Testing is also recommended with a family history of a parent with heritable pathogenic $11 \mathrm{p} 15$ anomaly, which places the child at a $50 \%$ risk of being affected. First tier testing is blood lymphocyte DNA

5 Hypoglycaemia is defined as plasma glucose levels $<50 \mathrm{mg} / \mathrm{dl}$ for the first 6 hours of life and $<60 \mathrm{mg} / \mathrm{dl}$ thereafter. Hyperinsulinism is defined as a glucose infusion rate of $\geq 8 \mathrm{mg} / \mathrm{kg} / \mathrm{min}$, a detectable level of insulin and/or C-peptide and undetectable levels of ketones and free fatty acids. Transient hypoglycaemia as a suggestive feature is defined by the above criteria lasting $<1$ week. Hyperinsulinism as a cardinal feature is defined by these criteria lasting $>1$ week and/or requiring escalated treatment

\section{BWS and ART}

6 There is an established association between ART and BWS. The absolute risk of BWS in an individual conceived by ART is estimated to be very low (no more than 1 in 1,000). Additional research is required to further characterize this association and the relationship between subfertility, hormonal stimulation, embryo manipulation and imprinting defects

ART, assisted reproduction technology; BWS, Beckwith-Wiedemann syndrome; BWSp, Beckwith-Wiedemann spectrum.

Features characterized as 'suggestive features' are likely to occur independently in the general paediatric population and are therefore given less weight in the consensus BWSp scoring system outlined in the next section. Suggestive features include a birthweight greater $>2$ standard deviation scores (SDS), facial naevus flammeus, polyhydramnios or placentomegaly, ear creases or pits, transient hypoglycaemia, embryonal tumours, nephromegaly or hepatomegaly and umbilical hernias or diastasis recti.

\section{Consensus scoring system and definition}

The many previously proposed systems to define BWS have suggested various combinations of clinical features (with macroglossia, exomphalos and/or (asymmetric) overgrowth as major features) ${ }^{11-13,18,21,22}$ with the aim of optimizing the likelihood of a classical and molecularly confirmed diagnosis. Frequently cited and recent phenotype articles from the past 25 years were reviewed for the prevalence of individual clinical features (Supplementary information S1 (table)), which were then classified as cardinal or suggestive features (BOX 2). As several of these articles referenced the same patient cohorts ${ }^{12-16,18,21-26}$, data were analysed from the nine articles describing apparently distinct cohorts ${ }^{11,12,14,15,18,21,23,25,26}$. The goal of this BWSp scoring system (TABLE 1; BOX 2) was to recognize that BWS falls into a clinical spectrum and that some features that have long been considered to be classical parts of the syndrome are not present in every patient, and therefore, the diagnosis should not be dismissed owing to the absence of such features. Additionally, this Consensus Statement sought to include elements that could be pathognomonic for BWS. In addition to informing the presence of a diagnosis of classical BWS, the consensus group also determined that the same system could be used to provide guidance regarding when to pursue genetic testing. We compared this new scoring system with previously published systems (Supplementary information S2 (figure)) while keeping in mind that previous systems focused on the diagnosis of classical BWS and molecularly confirmed BWS and not the diagnosis of the BWSp.

Cardinal features are considered key to the clinical diagnosis, whereas suggestive features add to the likelihood of a clinical diagnosis and the indications for molecular testing but are less specific (BOX 2). Cardinal and suggestive feature designations were analysed in the BWSp cohort reported by Ibrahim et al. ${ }^{11}$ and were shown to be largely superior to previous diagnostic systems (Supplementary information S2 (figure)). Limitations of the study were that transient hypoglycaemia versus prolonged hyperinsulinism were not typically distinguished in the prior cohorts, so this feature could therefore not be assessed, and that macrosomia was variably defined in previous cohorts ${ }^{11-14,17,18,21,22}$. 
BWSp

\begin{tabular}{|c|c|c|}
\hline $\begin{array}{c}\text { Isolated } \\
\text { lateralized } \\
\text { overgrowth }\end{array}$ & Atypical BWS & BWS \\
$\begin{array}{c}\text { Other } \\
\text { genetic } \\
\text { causes }\end{array}$ & $\begin{array}{c}11 \mathrm{p} 15 \text { imprinted } \\
\text { region dysregulation }\end{array}$ & $\begin{array}{c}\text { Clinical } \\
\text { BWS } \\
\text { diagnosis }\end{array}$ \\
diagnos
\end{tabular}

Figure 1 | The Beckwith-Wiedemann spectrum. The consensus group introduced the concept of the Beckwith-Wiedemann spectrum (BWSp), which includes patients with a clinical diagnosis of Beckwith-Wiedemann syndrome (BWS) with or without an (epi) genetic change at the BWS locus on chromosome 11p15, patients with 'atypical BWS' (defined as fewer cardinal and suggestive features than those needed for a clinical diagnosis of BWS) and an (epi)genetic change at the BWS locus, and patients with 'isolated lateralized overgrowth' and an (epi)genetic change at the BWS locus. The dotted arrow indicates that some patients with apparent isolated lateralized overgrowth and no 11p15 abnormality might subsequently be found to have an 11p15 abnormality on testing of additional tissues or with a more sensitive assay. Patients with clinical BWS and no detectable 11p15 abnormality might be further investigated with additional clinical evaluation and consideration of other syndromes, which may have features overlapping with BWSp, and appropriate testing for those syndromes may be warranted.

Cardinal features include macroglossia, exomphalos, lateralized overgrowth, multifocal Wilms tumour, prolonged hyperinsulinism and distinct pathologic findings that are unique to BWS. The major differences between the consensus and previous scoring systems are the classification of macrosomia and hyperinsulinism. Although it is often associated with BWSp, macrosomia (defined as height and/or weight $>2$ SDS) is no longer considered a cardinal feature, as it is variably defined in previous cohorts and it might be present in only approximately onehalf of patients with BWS ${ }^{16,26}$. Hyperinsulinism (defined in TABLE 1, R5) without another identifiable molecular cause can be the initial presenting feature of $\mathrm{BWS}^{27228}$. Hyperinsulinism is classified as a cardinal feature when lasting $>1$ week and requiring escalated treatment and as a suggestive feature when lasting $<1$ week.

For simplicity and consistency, we have developed consensus criteria by use of cardinal and suggestive features (TABLE 1, R2). For a clinical diagnosis of classical BWS, a patient requires a score of $\geq 4$ based on cardinal and suggestive features; this clinical diagnosis does not require the molecular confirmation of an 11p15.5 anomaly. Patients with a score of $\geq 2$ (including those with classical BWS with a score of $\geq 4$ ) merit genetic testing according to our algorithm for investigation and diagnosis of BWS (FIG. 2). Patients with a score of $<2$ do not meet the criteria for genetic testing. Patients with a score of $\geq 2$ with negative genetic testing should be considered for an alternative diagnosis and/or referral to a BWS expert for further evaluation.

Clinical diagnosis within the BWSp beyond the clear diagnosis of classical BWS or a clear molecular diagnosis is challenging and requires a combination of molecular testing and physician opinion. There is currently not enough published data to provide clear clinical recommendations for patients with a score of $<4$ who have no molecular abnormality. Nonetheless, patients with a cardinal feature of BWS (such as macroglossia, hyperinsulinism, a multifocal Wilms tumour or a pathological finding) should be referred to a specialist with expertise in BWS for further evaluation. Patients with isolated exomphalos are more common and are less likely to have an 11p15.5 defect compared with patients with other isolated symptoms, and should therefore not be included in the BWSp. Lateralized overgrowth can occur both as a symptom of BWSp and independent of BWSp ${ }^{10}$. When lateralized overgrowth occurs with an 11p15 abnormality, it is considered part of BWSp. As there are multiple molecular causes of lateralized overgrowth aside from $11 \mathrm{p}$ anomalies (for example, PIK3CA and AKT1 mutations), lateralized overgrowth without an $11 \mathrm{p} 15$ anomaly in a child who does not meet the criteria for classical BWS was considered to be outside the BWSp and the scope of this Consensus Statement; thus, recommendations for further investigation and clinical management were not made (TABLE 1, R3).

\section{Indications for molecular testing}

The consensus group recommended that molecular testing is indicated in patients with a score of $\geq 2$ (BOX 2) unless there is an alternative explanation (for example, gestational diabetes mellitus for macrosomia) (TABLE 1, R4). For isolated exomphalos, molecular testing is discretionary. Testing is recommended in patients with a family history and a known heritable pathogenic $11 \mathrm{p} 15$ anomaly (a positive family history might occur in $10-15 \%$ of patients ${ }^{2,29}$ ). Some features included in some previous diagnostic criteria (for example, cleft palate, advanced bone age, polydactyly and supernumerary nipples) are suggestive of an alternative diagnosis such as Simpson-Golabi-Behmel syndrome ${ }^{30}$ and are therefore not included in the consensus scoring system. Although renal abnormalities are common in patients with BWSp, they are usually present with other features and not as an isolated feature. When molecular testing is negative, other relevant disorders should be considered in the differential diagnosis (FIG. 2; see Supplementary information S3 (table)).

\section{Assisted reproduction technology}

Assisted reproductive technologies (ARTs) are defined as treatments handling male and female gametes outside of the body and include procedures such as in vitro fertilization (IVF) and intracytoplasmic sperm injection (ICSI) ${ }^{31}$. ARTs account for $1-3 \%$ of all births in industrialized countries $^{31}$. Although these techniques are regarded as safe, it has been suggested that the establishment and/or maintenance of DNA methylation at imprinted loci might be disturbed by ART ${ }^{31-36}$. Following reports of children with a rare molecular subtype of Angelman syndrome who were conceived by ICSI ${ }^{32,33}$ and an increased frequency ( 4-6-fold) of ART births in children with BWS ${ }^{34-36}$, a population-based study estimated the risk of BWS in IVF-conceived children to be approximately 1 in 4,000, substantially greater than that in the general population ${ }^{31}$. A study from 2017 reported a tenfold increased risk of BWS with ART but an absolute risk of approximately 1 in 1,000 (REF. 37). Although some epidemiological studies have not detected an increased relative risk of BWS in children born after $\mathrm{ART}^{38,39}$, molecular studies support an association, as $>90 \%$ of children with BWS conceived by 


\section{Box 2 | Clinical features of Beckwith-Wiedemann spectrum \\ Cardinal features ( 2 points per feature) \\ - Macroglossia \\ - Exomphalos \\ - Lateralized overgrowth \\ - Multifocal and/or bilateral Wilms tumour or nephroblastomatosis \\ - Hyperinsulinism (lasting >1 week and requiring escalated treatment) \\ - Pathology findings: adrenal cortex cytomegaly, placental mesenchymal dysplasia or pancreatic adenomatosis}

Suggestive features ( 1 point per feature)

- Birthweight $>2$ SDS above the mean

- Facial naevus simplex

- Polyhydramnios and/or placentomegaly

- Ear creases and/or pits

- Transient hypoglycaemia (lasting $<1$ week)

- Typical BWSp tumours (neuroblastoma, rhabdomyosarcoma, unilateral Wilms tumour, hepatoblastoma, adrenocortical carcinoma or phaeochromocytoma)

- Nephromegaly and/or hepatomegaly

- Umbilical hernia and/or diastasis recti

For a clinical diagnosis of classical Beckwith-Wiedemann syndrome (BWS), a patient requires a score of $\geq 4$ (this clinical diagnosis does not require the molecular confirmation of an 11p15 anomaly). Patients with a score of $\geq 2$ (including those with classical BWS with a score of $\geq 4$ ) merit genetic testing for investigation and diagnosis of BWS. Patients with a score of $<2$ do not meet the criteria for genetic testing. Patients with a score of $\geq 2$ with negative genetic testing should be considered for an alternative diagnosis and/or referral to a BWS expert for further evaluation. BWSp, Beckwith-Wiedemann spectrum; SDS, standard deviation scores.

ARTs have an epimutation at the centromeric imprinting centre KCNQ1OT1:transcriptional start site (TSS) differentially methylated region (DMR) (also known as IC2), compared with $\sim 50 \%$ of children with BWS who were not conceived by $\mathrm{ART}^{40}$. Various factors might contribute to an association between ARTs and Angelman syndrome or BWS, including infertility per se (that is, independently to ART techniques) ${ }^{41,42}$, superovulation or in vitro embryo culture ${ }^{43-45}$. The link between ART and epigenetic defects has also been suggested by the large offspring syndrome (an ART-associated phenomenon in sheep and cows that has some phenotypic similarity to BWS), which has been reported to be associated with epigenetic alterations similar to those observed in $\mathrm{BWS}^{46}$. However, although there is clear evidence linking ARTs and BWS, additional research is required to further elucidate the relationships between subfertility, hormonal stimulation, embryo manipulation and imprinting defects (TABLE 1, R6).

\section{Molecular aspects of BWSp}

BWSp is associated with molecular abnormalities affecting a cluster of imprinted genes located within the chromosome region 11p15.5-11p15.4, which is divided into two functionally independent domains: the centromeric and telomeric domains ${ }^{2}$ (FIG. 3). Each domain harbours its own imprinting control region, marked by a DMR. The insulin-like growth factor 2 (IGF2)-encoding gene (IGF2) and the gene encoding the non-translated long non-coding RNA (lncRNA) H19 are located in the telomeric domain and are controlled by the H19/IGF2: intergenic (IG) DMR (also known as imprinting control region 1 (ICR1), H19-DMR or Imprinting Centre 1 (IC1); OMIM *616186). The cell cycle inhibitor gene CDKN1C and the gene encoding the regulatory lncRNA KCNQ1OT1 are located in the centromeric domain and are controlled by the KCNQ1OT1:TSS DMR (also known as KvDMR, LIT1-DMR, imprinting control region 2 (ICR2) or Imprinting Centre 2 (IC2); OMIM *604115). The Human Genome Variation Society (HGVS)-recommended nomenclature, H19/IGF2:IG DMR and KCNQ1OT1:TSS DMR, should be adopted in publications and test reporting ${ }^{47}$ (TABLE 2, R7); however, for brevity, IC1 and IC2 are used hereafter.

A molecular defect affecting imprinted genes in chromosome region $11 \mathrm{p} 15$ can be demonstrated in $~ 80 \%$ of patients with BWSp2 ${ }^{2}$. DNA methylation abnormalities are the most frequent defects; loss of methylation (LOM) at the maternal IC2 allele is found in $~ 50 \%$ of patients and gain of methylation (GOM) at the maternal ICl allele in $5-10 \%$ of patients ${ }^{48}$. Mosaic, segmental paternal uniparental isodisomy (UPD) of 11p15.5 (commonly referred to as segmental upd(11)pat) can be detected in $20 \%$ of patients, intragenic $C D K N 1 C$ mutations can be detected in $5 \%$ of sporadic and $40 \%$ of familial cases, and chromosomal abnormalities in $11 \mathrm{p} 15$ can be detected in $<5 \%$ of patients; a molecular diagnosis is not reached in up to $20 \%$ of patients ${ }^{2}$. The frequency of twinning is markedly higher in patients with BWS than the general population; in the majority of cases, twins are female, monozygous and discordant (that is, one twin is affected and one is unaffected by BWSp $)^{49}$. Owing to the sharing of circulation during development, DNA from blood cells or saliva might show aberrant DNA methylation (usually IC2 LOM) in both affected and unaffected discordant twins, whereas methylation is concordant with phenotype in non-blood-derived samples such as buccal swab ${ }^{50}$. Thus, buccal swab is the preferred source of DNA for unambiguous diagnosis in cases of discordant monozygotic twins.

\section{Molecular genetic testing for BWSp}

A flowchart summarizing the molecular diagnostic pathway for investigation of suspected BWSp is presented in FIGURE 2 (TABLE 2, R8).

First-line molecular testing procedures should assay IC1 and IC2 methylation (TABLE 2, R9). Methylation is abnormal in cases of IC2 LOM, IC1 GOM, copy number variations (CNVs) and segmental upd(11)pat (both IC2 LOM and IC1 GOM) ${ }^{51}$. Abnormal methylation status confirms a diagnosis of BWSp, but its underlying mechanism must be established to define management, genetic counselling and recurrence risks ${ }^{48,51}$. Thus, if methylation is assayed using a technique that does not estimate DMR copy number, this should then be determined in all patients with IC1 and/or IC2 methylation abnormalities (FIG. 2). Currently, methylation-specific (MS) multiplex ligation-dependent probe amplification (MS-MLPA) is the most common diagnostic test, as it simultaneously detects DMR methylation status and copy number; however, other techniques (such as MS-PCR and MS quantitative PCR) are more sensitive in patients with low-level mosaicism (for a detailed list, see REFS 51-54 and the references within). 


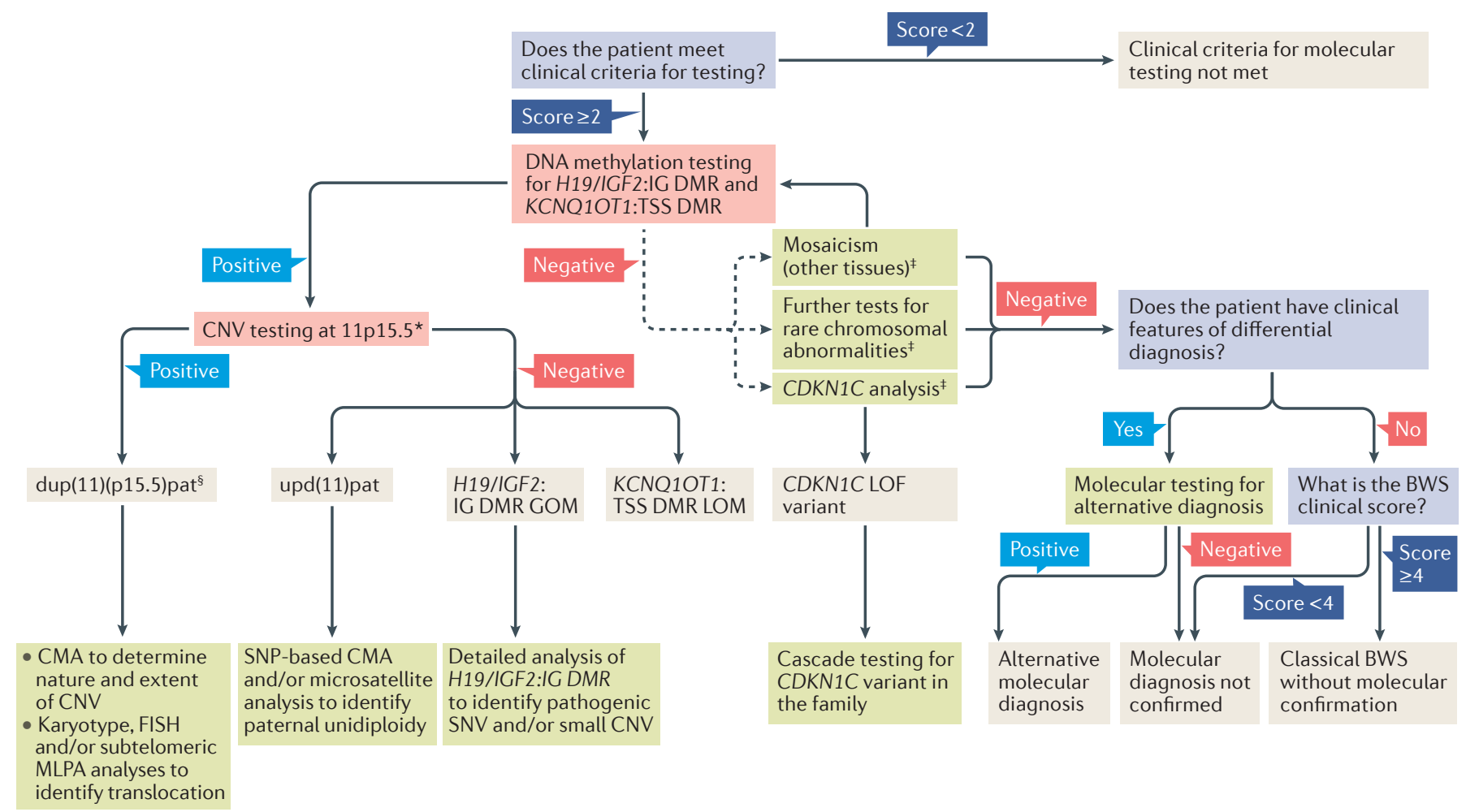

Clinical questions

Recommended molecular testing

Molecular diagnosis

Molecular testing to be considered

Figure 2 | Flowchart for investigation and diagnosis of BeckwithWiedemann syndrome. The figure summarizes the molecular diagnostic pathway for investigation of suspected Beckwith-Wiedemann spectrum (BWSp). Patients with clinical features reaching a score of $\geq 2$ should be genetically tested. It is recommended that first-line testing should assay the methylation status of H19/IGF2:intergenic (IG) differentially methylated region (DMR) (also known as IC1) and KCNQ1OT1:transcriptional start site (TSS) DMR (also known as IC2). If not estimated simultaneously with DNA methylation, DMR copy number should then be determined in all patients with IC1 and/or IC2 methylation abnormalities. These assays can yield positive molecular diagnosis of BWSp with IC2 loss of methylation (LOM), IC1 gain of methylation (GOM), segmental paternal uniparental isodisomy (UPD) of 11p15.5 (upd(11)pat) or copy number variation (CNV; most commonly duplication of paternal 11p15.5 (dup(11)(p15.5)pat)). Further molecular tests can be considered to determine underlying mechanism of methylation abnormality, UPD or CNV. If DNA methylation testing is negative, further molecular tests can be considered to identify mosaic methylation abnormalities, pathogenic CDKN1C variants or rare balanced chromosomal rearrangements. If all molecular tests are negative, differential diagnosis should be considered. However, a diagnosis of classical Beckwith-Wiedemann syndrome (BWS) is made in the presence of a clinical score of $\geq 4$ even in the absence of the molecular confirmation of an 11p15 anomaly. CMA, chromosome microarray analysis (can be oligonucleotide-based and/or single-nucleotide-polymorphism-based platforms); FISH, fluorescence in situ hybridization; LOF, loss of function; MLPA, multiplex ligation-dependent probe amplification; SNP, single nucleotide polymorphism; SNV, single nucleotide variation. ${ }^{*} \mathrm{CNV}$ status may be

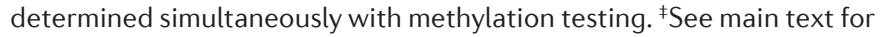
indications for testing. ${ }^{\S}$ Deletion of maternal 11p15.5 (del(11)(p15.5)mat) may be detected with lower frequency.
Recommended investigations if testing for methylation abnormalities is positive. If a DMR CNV is implied using a PCR-based methodology (such as MS-MLPA), chromosome microarray analysis (such as oligonucleotide polymorphism-based or single nucleotide polymorphism (SNP)-based arrays) should be considered to determine the nature and extent of the deletion or duplication, and karyotyping, fluorescence in situ hybridization or subtelomeric MLPA should be considered to identify possible chromosomal translocations ${ }^{54-57}$. Testing can then be extended to other family members as appropriate. A SNP-based array will also allow detection of uniparental disomy (and indeed mosaicism) if a CNV is not detected.
If IC1 GOM and IC2 LOM are detected without evidence of CNV, mosaic segmental upd(11)pat is probable and can, if necessary, be confirmed using microsatellite analysis or SNP-based chromosome microarray analysi $^{58-61}$. SNP-based chromosome microarray is considered to be the most sensitive method to investigate low-level mosaic (for example, 1-5\%) segmental upd(11)pat ${ }^{59}$. Mosaic paternal unidiploidy (that is, genome-wide paternal UPD) affects up to $10 \%$ of patients with uniparental disomy and, as mosaic paternal unidiploidy is associated with additional clinical features and an increased risk of tumour development, further investigations (SNP array or microsatellite analysis) to detect this molecular abnormality should be considered ${ }^{62-67}$ (TABLE 2, R10). 

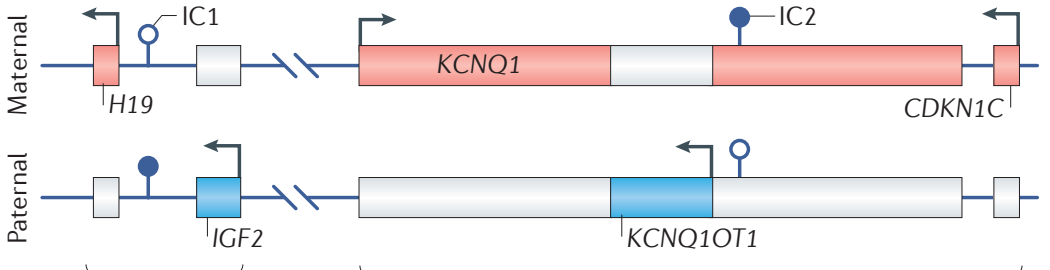

Telomeric domain

Centromeric domain

Figure 3 | The Beckwith-Wiedemann syndrome locus at chromosome 11p15.5. The figure depicts the chromosome 11p15.5-11p15.4 region with the imprinted genes and control regions that are implicated in the pathophysiology of Beckwith-Wiedemann spectrum (BWSp). The BWSp locus can be divided into two functionally independent domains, the telomeric and centromeric domains. Each domain harbours its own imprinting control region that is differentially methylated on the maternal and paternal chromosomes. The insulin-like growth factor 2 (IGF2)-encoding gene (IGF2) and the gene encoding the non-translated long non-coding RNA H19 are located in the telomeric domain and are controlled by the H19/IGF2:intergenic (IG) differentially methylated region (DMR) (also known as IC1), which is methylated on the paternal chromosome. The cell cycle inhibitor gene $C D K N 1 C$ and the gene encoding the regulatory long non-coding RNA KCNQ1OT1 are located in the centromeric domain and are controlled by the KCNQ1OT1:transcriptional start site (TSS) DMR (also known as IC2), which is methylated on the maternal chromosome. Genes expressed from the maternal chromosome are depicted as red boxes, and genes expressed from the paternal chromosome are depicted as blue boxes. Grey boxes indicate non-expressed alleles. Filled lollipops indicate methylated imprinting centres (ICs), and open lollipops indicate unmethylated ICs. Bent arrows indicate the orientation of transcription.

Up to $20 \%$ of patients with IC1 GOM might carry small CNVs in the DMR, which cannot be detected using chromosome microarray analysis, or single nucleotide variations (SNVs) in octamer-binding protein 4 (OCT4; also known as POU domain, class 5, transcription factor 1) or transcription factor SOX binding sites; these CNVs and SNVs are associated with a high risk of recurrence ${ }^{68-74}$. Although some small CNVs can be detected using MS-MLPA, detection of SNVs would require additional investigations that are unavailable in most diagnostic laboratories (TABLE 2, R11). However, targeted IC1 sequencing can be considered in a specialized laboratory if MS-MLPA shows IC1 GOM and no $\mathrm{CNV}$, especially if there is a family history of BWSp.

IC2 LOM is the most common epigenetic finding in BWSp, but IC2 DMR deletions are rare ${ }^{55}$, and at present, there is no indication for analysing patients with BWSp and IC2 LOM for SNVs. Approximately one-third of patients with IC2 LOM have multilocus imprinting disturbance (MLID) $)^{75-78}$. In most patients, the clinical importance of MLID is uncertain, and therefore, routine testing for MLID is not usually indicated; however, in patients with IC2 LOM and a family history of BWSp and no IC2 DMR CNV, MLID testing might help to determine if further testing for trans-acting mutations should be considered ${ }^{79,80}$.

Recommended investigations iffirst-line molecular testing is negative. A negative result for first-line molecular testing for IC1 and IC2 methylation does not exclude BWSp for a variety of reasons, including the possibility of low-level mosaicism that is below the limit of detection of methylation testing, a $C D K N 1 C$ mutation, a rare balanced chromosomal rearrangement (for example, inversion and/or translocation), an unrecognized or undetected cause of BWSp ( 20\% of patients with a characteristic BWS phenotype remain without a molecular diagnosis ${ }^{2}$ ) or an incorrect clinical diagnosis (TABLE 2, R12-15). Further molecular testing should be prioritized according to the most probable cause; for example, a less severe phenotype with lateralized overgrowth would suggest mosaicism, whereas a classical BWS phenotype with an abdominal wall defect and a positive family history would indicate a potential CDKN1C mutation.

Mosaicism for the molecular defect occurs in most sporadic cases of BWSp, and different tissues might have different proportions of affected cells ${ }^{81}$. First-line diagnostic testing is usually performed using blood-leukocyte DNA, and IC1 or IC2 methylation level might be equivocal or within the normal range. Analysis of DNA from buccal swabs, cultures of fibroblasts or cells of mesenchymal origin (for example, obtained from surgical resection and/or excision of hyperplastic tissues) improve the detection rate for all mosaic defects ${ }^{51,54,81}$ (TABLE 2, R13).

CDKN1C mutations account for $\sim 5 \%$ of sporadic cases of BWS and for $40 \%$ of familial cases (in the case of maternal inheritance $)^{48,82}$. Detection of a candidate pathogenic CDKN1C variant enables appropriate cascade testing to clarify familial recurrence risks ${ }^{82,83}$. Rare cases of apparently de novo CDKN1C mutations can arise in siblings, presumably resulting from maternal germline mosaicism $^{83}$.

Rare maternally inherited balanced translocations or inversions involving chromosome region $11 \mathrm{p} 15$ might or might not be associated with IC2 methylation anomalies and should be considered if first-line testing is negative ${ }^{84-87}$ (TABLE 2, R14). Finally, when molecular testing is negative, other relevant disorders should be considered as differential diagnoses (FIG. 2; TABLE 2, R15; see Supplementary information S3 (table)).

\section{Multilocus imprinting disturbance}

MLIDs are those with altered DNA methylation in addition to the lesion responsible for the primary clinical presentation $^{88}$. MLID has a higher prevalence in BWSp than in other imprinting disorders, and genome-wide analyses have revealed MLID in approximately one-third of patients with BWSp who have IC2 LOM but not in patients with segmental upd(11)pat or IC1 GOM ${ }^{48,75-78,81,88-91}$. In BWSp, MLID almost exclusively involves loci methylated in the maternal, and not the paternal, germ line, although rare cases show LOM at both IC2 and IC1 (the latter finding is a feature of the growth restriction disorder Silver-Russell syndrome (SRS) $)^{76,92}$.

Rare cases of BWSp-MLID have been associated with biallelic maternal-effect genetic mutations in NLRP2 (REF. 79) and NLRP5 (REF. 80) (which encode NACHT, LRR and PYD domains-containing protein 2 (NLRP2) and NLRP5), and therefore, the possibility of an underlying trans-acting genetic mutation in these genes may warrant consideration in genetic counselling.

Perhaps owing to the various methylation alterations and frequent mosaicism seen in MLID, its effect on the BWS clinical phenotype remains unclear ${ }^{75,76,78,89,92,93}$, and therefore, routine clinical diagnostic testing is not a recommendation of this consensus. 
Table 2 | Consensus recommendations of the molecular working group

\section{Molecular genetic analysis}

$7 \quad$ Molecular genetic testing should be performed by a health professional experienced in the field of imprinting disorders. Recommended nomenclature (for example, HGVS) should be adopted in publications and in test reporting

8 The flowchart outlined in FIG. 2 should be followed for molecular diagnosis of BWSp

9 First-line molecular testing should include DNA methylation analysis of the H19/IGF2:IG DMR (IC1) and KCNQ1OT1:TSS DMR (IC2) regions. If a DNA methylation defect at either or both DMRs is found, further tests should be performed to identify possible underlying CNV or upd(11)pat (if it was not discriminated in initial diagnostic testing)

10 Given the different tumour spectrum associated with mosaic genome-wide paternal uniparental disomy, further testing should be considered to distinguish this condition from upd(11)pat

11 Detailed analysis of the H19/IGF2:IG DMR should be considered in individuals with GOM of this region, as SNVs and/or small CNVs can occur in these patients and confer high risk of recurrence (prioritized in the presence of a positive family history)

12 In patients with a negative methylation test result, second-line molecular testing should be considered and might include sequencing of the coding exons and the exon-intron boundaries of CDKN1C (prioritized in the presence of a positive family history, a cleft palate or an abdominal wall defect (umbilical hernia or exomphalos))

13 In patients with a negative methylation test result, second-line molecular testing should be considered and might include analysis of additional tissues to detect somatic mosaicism (prioritized in the presence of asymmetric overgrowth)

14 In patients with a negative methylation test result, second-line molecular testing should be considered and might include further tests for rare chromosomal rearrangements

15 In patients with a negative methylation test result, second-line molecular testing should be considered, and might include re-evaluation of the clinical diagnosis and reconsideration of differential diagnoses

16 Genetic counselling should be performed by a health professional experienced in the field of imprinting disorders

17 As the recurrence risk associated with genetic defects (for example, CDKN1C loss of function variants, CNVs and DMR $\mathrm{SNV}$ ) is dependent on their size, location and parental origin, these factors should be taken into consideration during counselling for the family

\section{Prenatal molecular genetic analysis}

18 Prenatal molecular diagnostic investigations should be considered if prenatal ultrasonography reveals potential features of BWSp and should lead to a specific diagnosis (or exclude other potential conditions); they should also be considered if a positive family history with a known molecular defect is present, which would influence the management of the relevant pregnancy

19 The flowchart indicated for postnatal testing (FIG. 2) is not necessarily applicable to prenatal testing. Modification of this flowchart depends on the individual setting (for example, known molecular defects and specific clinical features)

20 Prior to offering prenatal diagnosis for BWSp, a detailed discussion of the technological limitations and ethical issues should be undertaken with the parents; in particular, they should be made aware that a normal result does not necessarily exclude the diagnosis

21 It is recommended that centres offering prenatal diagnosis prospectively collect information on the true/false positive/ negative diagnostic rates and that this information is contributed to multicentre audits to enable best practice guidelines to be further developed and refined

BWSp, Beckwith-Wiedemann spectrum; CNV, copy number variation; DMR, differentially methylated region; GOM, gain of methylation; HGVS, Human Genome Variation Society; SNV, single nucleotide variant; upd(11)pat, segmental paternal uniparental isodisomy of 11p15.5.

\section{Recurrence risks in BWSp}

The risk of recurrence for BWSp depends on the genetic aetiology and nature of any genetic or epigenetic defect identified as well as its parental origin. It is therefore recommended that family counselling should be performed by an individual experienced in the field of imprinting disorders and should take into account the precise nature of the anomaly detected (TABLE 2, R16,17).

It has been reported that up to $10-15 \%$ of cases of BWSp are familial and most commonly result from CDKN1C mutations, chromosome 11p15 abnormalities and genetic alterations within IC1 (REFS 2,29). In these cases, the mode of inheritance is autosomal dominant, but the recurrence risk is dependent on the sex of the parent transmitting the affected allele (TABLE 2, R17; TABLE 3).
Pathogenic CDKN1C variations have a $50 \%$ recurrence risk with variable expressivity if the mutation is inherited from the mother ${ }^{82,83}$. In principle, all $11 \mathrm{p} 15$ CNVs and balanced translocations have a 50\% recurrence risk with phenotypes dependent on the parental origin ${ }^{54,84,86,87}$. In cases with paternal duplication of 11 p15 resulting from the unbalanced segregation of a translocation or an inversion, individuals carrying the balanced rearrangement have a normal phenotype ${ }^{94}$. In some pedigrees, either BWSp or SRS has been observed depending upon paternal or maternal transmission of the 11 p15 duplication ${ }^{57,95,96}$. Paternal transmission of a duplicated telomeric domain and maternal transmission of a deleted centromeric domain also usually result in BWSp with a high recurrence risk $^{52,95,97,98}$. Prediction of 
Table 3 | Summary of Beckwith-Wiedemann spectrum molecular defect categories and recurrence risk

\begin{tabular}{|c|c|c|c|c|}
\hline $\begin{array}{l}\text { Molecular } \\
\text { defect }\end{array}$ & $\begin{array}{l}\text { Frequency of } \\
\text { molecular defect }\end{array}$ & $\begin{array}{l}\text { Mosaicism } \\
\text { observed }\end{array}$ & Risk of recurrence & $\begin{array}{l}\text { Characteristic clinical features (compared } \\
\text { with other molecular subgroups) }\end{array}$ \\
\hline IC1 GOM & $5 \%{ }^{48}$ & Yes $^{27,54,76,78,81}$ & $\begin{array}{l}\text { - If no genetic anomaly is present, }<1 \% 2 \\
\text { - If genetic anomaly (for example, } \\
\text { pathogenic SNV of copy number } \\
\text { variant in the DMR) is present, } 50 \% \text {; } \\
\text { dependent on parental origin }\end{array}$ & $\begin{array}{l}\text { - Low frequency of exomphalos }{ }^{11,14,17} \\
\text { - High risk of Wilms tumour }{ }^{14,58,149}\end{array}$ \\
\hline IC2 LOM & $50 \%{ }^{48}$ & $Y^{2} s^{27,54,76,78,81}$ & $\begin{array}{l}\text { - If no genetic anomaly is identified, }<1 \%^{2} \\
\text { - If a cis-acting genetic anomaly is } \\
\text { present, } 50 \% \text {; dependent on parental } \\
\text { origin }^{9-103}\end{array}$ & $\begin{array}{l}\text { - High frequency of exomphalos }{ }^{11,14,17} \\
\text { - Low risk of Wilms tumour }{ }^{14,58,149}\end{array}$ \\
\hline upd(11)pat & $\begin{array}{l}20 \%^{48} \text { (see } \\
\text { also paternal } \\
\text { unidiploidy) }\end{array}$ & $Y^{27,54,61,76,78,81}$ & $<1 \%^{2}$ & $\begin{array}{l}\text { - High incidence of lateralized overgrowth } \\
\text { - Low frequency of exomphalos }{ }^{11,14,17} \\
\text { - High risk of Wilms tumour and } \\
\text { hepatoblastoma } \\
\text { hi,58,149 }^{114}\end{array}$ \\
\hline $\begin{array}{l}\text { Loss-of-function } \\
\text { CDKN1C } \\
\text { variants }\end{array}$ & 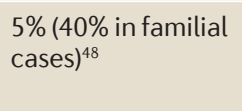 & $\begin{array}{l}\text { Usually no } \\
\text { but has been } \\
\text { reported rarely }\end{array}$ & $50 \%$ on maternal transmission ${ }^{82,83}$ & $\begin{array}{l}\text { - High frequency of exomphalos }{ }^{11,14,17} \\
\text { - Low risk of Wilms tumour }{ }^{14,58,149}\end{array}$ \\
\hline $\begin{array}{l}\text { Deletions } \\
\text { involving } 11 p 15\end{array}$ & $1-2 \%^{55,98}$ & $\mathrm{No}^{55}$ & $\begin{array}{l}\text { Dependent on extent and position of } \\
\mathrm{CNV} \text {, and parent of origin }{ }^{55}\end{array}$ & - \\
\hline $\begin{array}{l}\text { Mosaic paternal } \\
\text { unidiploidy } \\
\text { (genome-wide } \\
\text { paternal UPD) }\end{array}$ & $\begin{array}{l}\text { Up to } 10 \% \text { of } \\
\text { upd(11)pat }{ }^{62-67,184}\end{array}$ & Yes $^{62-67,184}$ & Low $^{62-67,184}$ & High frequency of neoplasia ${ }^{63,64,137,152}$ \\
\hline MLID & $\begin{array}{l}\text { 33\% of IC2 LOM } \\
\text { cases }^{75-78,88}\end{array}$ & Yes $^{78,88-91}$ & $\begin{array}{l}\text { Low unless an in trans genetic variant is } \\
\text { identified }\end{array}$ & Unclear $^{75,76,78,89,92,93}$ \\
\hline
\end{tabular}

DMR, differentially methylated region; dup(11)(p15.5)pat, duplication of paternal 11p15.5; GOM, gain of methylation; LOM, loss of methylation; MLID, multilocus imprinting disturbance; SNV, single nucleotide variation; UPD, uniparental isodisomy; upd(11)pat, paternal uniparental isodisomy of $11 \mathrm{p} 15.5$.

phenotypes and recurrence risks that are associated with smaller CNVs within either the telomeric or centromeric domain can be complex, as it depends on their size and the genes and regulatory elements involved ${ }^{55,99-104}$.

Internal $\mathrm{IC} 1 \mathrm{CNV}$ s and SNVs have recurrence risk as high as $50 \%$ when occurring on the maternal allele, although incomplete penetrance and possible anticipation have been observed in some cases ${ }^{73,105,106}$. Rare familial cases of BWSp-MLID might be caused by maternal-effect gene mutations (for example, NLRP2 or NLRP5 mutations) and might be associated with a very high recurrence risk ${ }^{79,80}$. In the patients that harbour other molecular defects, the recurrence risk is generally low (TABLE 3).

\section{Prenatal molecular diagnosis}

Prenatal testing for BWSp poses particular challenges because, in addition to general aspects of molecular testing (such as the range of molecular disturbances, the challenge of mosaicism detection and the technical limitations of testing), the reliability and informative value of prenatal test results and the ethical issues involved must be considered before sampling ${ }^{107}$.

The major indications for prenatal diagnosis of BWSp are familial cases with a known genetic alteration and a high recurrence risk and patients with no family history in which possible features of BWSp (usually exomphalos but also macrosomia, hemihypertrophy, organomegaly and polyhydramnios) have been detected using prenatal fetal ultrasonography (TABLE 2, R18). The diagnostic testing for prenatal samples does not therefore necessarily follow the same flow as that for postnatal samples but reflects the individual situation (TABLE 2, R19).

Although chorionic villus (CVS) cells, amniotic fluid cells or fetal blood cells (native and cultured) might be used for molecular testing, it is possible that cell culture might influence the methylation patterns ${ }^{108}$. In CVS cells, the methylation pattern at 11p15.5 might be different from that of embryonic tissues ${ }^{108}$, and/or CVS cells might not reflect the (epi)genetic constitution of the fetus, and therefore, false-positive results might occur ${ }^{107}$. False-negative prenatal test results might occur with all types of testing owing to mosaicism; therefore, a normal prenatal test result cannot absolutely exclude a diagnosis of BWSp (TABLE 2, R20). Because of the fairly recent adoption of prenatal testing and the challenges involved, it is recommended that multicentre audit of cases, methods and diagnostic rates be implemented prospectively, to enable ongoing refinement of best practice guidelines (TABLE 2, R21).

\section{Care and management aspects of BWSp}

In view of the complex multisystem manifestations of BWSp, the consensus group recognized the requirement for effective coordination of health care (TABLE 4, R22). 
Table 4 | Recommendations of the management working group

\section{R Recommendation}

Strength of recommendation

22 It is recommended that each patient with BWSp should have an experienced lead health-care provider who will organize A+++ the referral to each specialist and will coordinate care for the patient

\section{Prenatal management}

23 If a diagnosis of BWSp is suspected or confirmed in the prenatal period, then potential BWSp-related fetal and maternal complications (for example, fetal congenital anomalies, shoulder dystocia from macrosomia, postnatal hypoglycaemia and maternal preeclampsia) should be anticipated and appropriate clinical care should be performed

24 If a diagnosis of BWSp is suspected or confirmed in the prenatal period, then delivery should take place in a clinical facility where neonatal intensive care can be provided

\section{Growth and lateralized overgrowth}

A+++

A+++

25 Growth charts from BWSp patients are needed

26 Physicians should be aware of the rare possibility of final height $>2$ SDS above the mean. Postnatal growth and pubertal development should be monitored at least annually until the end of growth

27 Appropriate interventions might be proposed in the case of possible tall stature with the same procedures as for other patients with tall stature

28 Monitoring of leg length discrepancy should be based on clinical examination

Patients with BWSp should be monitored for LLD at least annually during childhood and referred to a paediatric orthopaedic A+++ (LO) surgeon if LLD is present

30 Shoe-lifts might be indicated for LLD $<2 \mathrm{~cm}$. Epiphysiodesis is usually indicated for predicted LLD $>2 \mathrm{~cm}$. Reversible

A++ (LO) epiphysiodesis might be preferred

31 Lengthening of the shorter normal limb should be considered only for specific cases

A+++ (LO)

32 Surgical correction of asymmetric overgrowth of the upper limbs is generally not indicated

A+++

A++

A++

$\mathrm{A}++(\mathrm{LO})$

$\mathrm{A}+++(\mathrm{LO})$

Management of macroglossia

33 If substantial airway obstruction is suspected, a careful evaluation including sleep studies (polysomnography) and/or pulmonologist consultation and ear, nose and throat consultation should be performed

34 Tongue reduction surgery should be considered usually after the age of 1 year if there are macroglossia-associated feeding problems, persistent drooling, speech difficulties, dental malocclusion and psychosocial problems caused by the altered appearance

35 Surgical intervention (adenoid tonsillectomy with or without tongue reduction surgery) should be considered earlier in cases of severe airway obstruction

36 In cases of feeding difficulties, support from feeding specialists and dietetics should be proposed

37 Tongue reduction surgery should be performed by an experienced surgical team after detailed assessment by a multidisciplinary team (including paediatric anaesthesiologists, intensive care unit members, surgeons, speech therapists and orthodontists), preferably in a reference centre

38 The results of surgery should be carefully audited, and postoperative follow-up should continue until age 16 years

Management of exomphalos

39 Treatment of exomphalos in the context of BWSp should be in accordance with general recommendations for the treatment of exomphalos; however, in BWSp-associated cases, attention should be paid to the risk of hypoglycaemia and the anaesthetic risks associated with severe macroglossia

\section{Management of hypoglycaemia}

40 Capillary blood glucose should be monitored in neonates with a clinical suspicion or confirmed diagnosis of BWSp for the first 48 hours of life. Hypoglycaemia should be defined by two consecutive ( $30 \mathrm{~min})$ glucose levels $<50 \mathrm{mg} / \mathrm{dl}(<2.75 \mathrm{mmol} / \mathrm{l})$ during the first 6 hours of life or $<60 \mathrm{mg} / \mathrm{dl}(<3.5 \mathrm{mmol} / \mathrm{l})$ later. In case of hypoglycaemia, the newborn baby should be transferred to a neonatal intensive care unit

41 A diagnostic fasting test (including measurement of glucose, insulin and ketones after 6 hours of fasting for full-term babies and after 4 hours for preterm babies) should be performed 48 hours after birth and before discharge from the nursery for neonates with a suspicion of BWSp

42 No specific management of hyperinsulinism and/or hypoglycaemia has been proposed in the context of BWSp, and management of hyperinsulinism and/or hypoglycaemia should be performed according to general recommendations

43 In case of severe persistent hyperinsulinism in a patient with BWSp, additional causes of hyperinsulinism should be investigated

\section{Management of cardiac lesions}

44 Physicians should be aware of the increased prevalence of cardiac anomalies in children with BWSp

45 A baseline, clinical cardiovascular examination should be performed at diagnosis in all children with clinical and/or molecular diagnosis of BWSp. Individuals with clinically detected or suspected cardiovascular abnormalities should be referred for specialist cardiac assessment and echocardiography

\section{A++}

A++

A++

A+++

A+++ 
Table 4 (cont.) | Recommendations of the management working group

\section{R Recommendation}

\section{Management of cardiac lesions (cont.)}

46 Annual evaluation and electrocardiogram are recommended in patients with genomic rearrangements involving the IC2 region B+

47 Management and follow-up of congenital cardiac lesions (for example, ventricular septal defect) should be as in the A+++ population without BWSp

\section{Management of neurological features}

48 Cognitive development should be monitored by the paediatrician. Particular attention should be paid to those with risk factors such as preterm birth and neonatal hypoglycaemia and to carriers of chromosome rearrangements or paternal genome-wide UPD

49 For patients with a clinical diagnosis of BWSp and a learning disability with no molecular or chromosomal anomaly, other potential diagnoses should be considered and excluded (Supplementary information S3 (table))

50 Neurological investigations, including MRI, might be indicated only in children with neurological symptoms

\section{Management of renal complications}

51 At diagnosis of BWSp, all patients should be screened for nephro-urological malformations by clinical evaluation and USS

52 Physicians should be aware of the possibility of hypercalciuria, which can lead to nephrocalcinosis

53 Patients with USS-detected anomalies should be referred to a paediatric nephrologist and urologist for specific follow-up

54 For patients undergoing abdominal surveillance for tumour screening, physicians and radiologists should pay attention to the possibility of nephrocalcinosis and/or stones

55 For patients with BWSp, at the time of adult transition, a nephro-urological evaluation (clinical examination, blood pressure A++ and USS) should be performed

\section{BWSp and embryonal tumours}

56 Screening should be stratified according to the genotype

57 Abdominal USS for BWSp-related tumours every 3 months until age 7 years is recommended for all patients with BWSp except patients with isolated IC2 LOM

58 For patients with BWSp and upd(11)pat, abdominal USS for Wilms tumour and hepatoblastoma every 3 months until age 7 years is recommended

59 For patients with BWSp and IC1 GOM, abdominal USS for Wilms tumour every 3 months until age 7 years is recommended

60 For patients with BWSp and IC2 LOM, no tumour surveillance is recommended

A+++

A+++

$\mathrm{A}++$

61 For patients with BWSp and a CDKN1C mutation, abdominal USS for neuroblastoma every 3 months until age 7 years is recommended

62 For patients with BWSp and an 11p15 duplication, abdominal USS for Wilms tumour every 3 months until age 7 years is recommended

63 For patients with classical BWS without a molecular defect, abdominal USS every 3 months until age 7 years is recommended

64 a-Fetoprotein (AFP) screening is not recommended for patients with BWSp

65 Catecholamine screening is not recommended for patients with BWSp

A+++

A++

A+++

A+++

66 There should be a lower threshold for investigation in cases of possible tumour-related symptoms or in response to parental concerns

67 Treatment of tumours in patients with BWSp might be different from treatment of patients with sporadic diseases and should be discussed with respective study groups unless specific BWSp recommendations are given in the relevant tumour treatment protocols

\section{Late-onset complications}

68 Individuals with BWSp should be reviewed at age 16-18 years to identify any complications that will require continued follow-up by adult health-care services

69 Young adults with BWSp should be alerted to the availability of genetic counselling so that they can seek advice prior to starting a family

70 Given the paucity of data on the long-term health effects of a diagnosis of BWSp, further research should be undertaken

\section{Psychological and counselling aspects}

71 Health professionals caring for children and families with BWSp should take a holistic approach to care and be prepared to offer referral to specialist counselling and family support services as required. Especially, psychological evaluation and support should be offered to children and their families if required

72 When the clinical diagnosis is confirmed, parents should be offered the contact details of BWSp support groups

A+++

A++

A+++

A+++

${ }^{\star} \mathrm{A} / \mathrm{B}+$

$\mathrm{A}+$

A+++

A++

A+

A+++

A+++

A+++

BWSp, Beckwith-Wiedemann spectrum; GOM, gain of methylation; LLD, leg length discrepancy; LO, lateralized overgrowth; LOM, loss of methylation; SDS, standard deviation scores; UPD, uniparental isodisomy; upd(11)pat, paternal uniparental isodisomy of 11p15.5; USS, ultrasound scan. *Equal numbers of participants chose option A and option B. 


\section{Prenatal management}

In patients with BWSp for whom a risk of recurrence has been identified (TABLE 3), some parents might wish to consider prenatal diagnosis. If a molecular diagnosis is not available or indicated, then ultrasonographic detection of an anterior abdominal wall defect or macroglossia or, less specifically, macrosomia, visceromegaly, polyhydramnios, placentomegaly or pancreatic overgrowth might indicate a likely diagnosis of $\mathrm{BWS}^{109}$. Rarer manifestations detectable by prenatal ultrasound scan (USS) include placental mesenchymal dysplasia, urinary tract abnormalities, cardiac defects, adrenal cysts and masses ${ }^{10,111}$. Abnormal prenatal biochemical screening results - for example, elevated levels of free $\beta$-human chorionic gonadotropin in the first trimester ${ }^{109-111}$ and/or increased $\alpha$-fetoprotein (AFP) levels in the second trimester (associated with exomphalos) ${ }^{112}$ - can be associated with BWSp in the fetus. In pregnancies known to be at increased risk of BWSp, the presence of a single anomaly (for example, exomphalos) might be sufficient to make a presumptive diagnosis.

In pregnancies without a previous history of BWSp, none of the prenatally detectable features of BWSp are, in isolation, pathognomonic. Approximately $10-20 \%$ of fetuses with a prenatally diagnosed isolated exompha$\operatorname{los}^{112,113}$ and $\sim 20 \%$ of those with placental mesenchymal dysplasia ${ }^{114}$ will have BWSp. As cytogenetic and/or chromosome microarray analysis are indicated for both these findings, molecular analysis for BWSp can also be performed on the same sample, and confirmation or exclusion of BWSp might be helpful for the parents. For less-specific features (for example, urinary tract abnormalities and cardiac defects), testing for BWSp is likely to depend on whether there are multiple BWSp-related features present.

When a prenatal diagnosis of BWSp is suspected or confirmed, the management of individual congenital anomalies (for example, exomphalos or cardiac defect) generally follows standard protocols based on usual local practices. However, macrosomia might cause problems (for example, shoulder dystocia) at delivery, and therefore, growth should be carefully monitored in the latter stages of pregnancy and appropriate arrangements for delivery should be made (TABLE 4, R23,24). BWSp is also associated with polyhydramnios and premature birth. Potential post-delivery complications such as neonatal hypoglycaemia, respiratory obstruction from macroglossia, surgical repair of exomphalos and so on should be anticipated, and appropriate monitoring and facilities should be put in place.

Maternal complications associated with a diagnosis of fetal BWSp include gestational hypertension ( 2.4-fold increased risk) and pre-eclampsia ${ }^{115,116}$. In addition, haemolysis, elevated liver enzymes and low platelets (HELLP) syndrome has been reported occasionally in BWS ${ }^{116,117}$. Therefore, in cases of a suspicion of fetal BWS, such a condition should be looked for with a lower threshold than with normal pregnancies.

\section{Growth and lateralized overgrowth}

Although prenatal and postnatal overgrowth have been considered to be cardinal features in previous reports, overgrowth occurs in only $43-65 \%$ of patients ${ }^{118}$. Overgrowth at birth might be relatively more common in patients with IC1 GOM and segmental pat(11)upd than in other molecular subgroups ${ }^{14,17}$. Postnatal growth is generally in the upper part of the normal range but usually slows in late childhood, and differences in growth trajectories between children with BWSp and those without it should be considered when making predictions of adult height. However, growth trajectories have not been well reported in patients with BWSp, and specific growth charts are needed (TABLE 4, R25). Although few data on final adult height are available, one study reported that final adult height was higher than parental target height, with a mean distance to target height of $1.7 \pm 1.1$ SDS and approximately one-half of the patients having height SDS >2 (REF. 15) (TABLE 4, R26). Advanced bone age is infrequent $(\sim 3 \%)^{119,120}$, and to date, there are no data regarding treatment of tall stature in cohorts of patients with BWSp (TABLE 4, R27).

Lateralized overgrowth might occur in all molecular subtypes of BWSp, but it is rare in patients with a CDKN1C mutation and is the most frequent feature in patients with segmental upd(11)pat ${ }^{11,14}$. Molecular abnormalities in region 11 p15 might be observed in patients with isolated lateralized overgrowth, which enables a diagnosis of BWSp in such cases ${ }^{119,121}$. Leg length discrepancy (LLD) can be associated with substantial morbidity and can negatively influence quality of life ${ }^{122}$. The management of LLD will depend on severity (TABLE 4, R28-31). Shoe-lifts might be indicated for LLD $<2 \mathrm{~cm}$. As in isolated (non-BWSp) LLD, epiphysiodesis might be considered for LLD discrepancy $>2 \mathrm{~cm}$ (REF. 123) (TABLE 4 , R30). Surgical correction of asymmetric overgrowth of the upper limbs is generally not indicated (TABLE 4, R32).

\section{Management of macroglossia}

Of children diagnosed with classical BWS, 90\% have macroglossia, and BWSp is the most common cause of macroglossia in childhood ${ }^{124}$. Although macroglossia might appear to regress spontaneously in some children (from a combination of a decrease in growth velocity and an increase in growth of the mandible), $40 \%$ of children with BWS undergo a surgical tongue reduction ${ }^{125}$. The most common indications for surgery are problems with feeding; persistent drooling; difficulties with articulation; orthodontic problems, including prognathism and development of an anterior open-bite and incisor spacing and/or flaring; and psychosocial difficulties resulting from abnormal cosmetic appearance and difficulties with speech, feeding and drooling ${ }^{126-128}$ (TABLE 4, R33-35).

The enlarged tongue is usually increased in size in all three dimensions, and the aim of surgery is to reduce the tongue bulk while preserving normal shape and improving function. The most common surgical approach is anterior wedge resection, but a variety of other techniques have been described ${ }^{125,128,129}$. Surgical complications, although infrequent, can include postoperative oedema of the tongue and wound dehiscence.

In rare cases, respiratory problems might require surgery to be performed in the neonatal period, and preoperative tracheostomy might be required ${ }^{125}$. When obstructive sleep apnoea is suspected, an airway evaluation and 
appropriate further investigation with polysomnography can be used for objective assessment ${ }^{130,131}$ (TABLE 4, $\mathrm{R} 33$ ). In the absence of respiratory obstruction, surgery is generally delayed until at least age 12 months (when tongue size is more stable) (TABLE 4, R34). If the indication for surgery is unclear, the child's progress should be monitored to determine whether indications arise in the future. Long-term follow-up studies generally show favourable results of surgery in most cases, with cosmetic improvement, reduced drooling, resolution of feeding difficulties, improved speech, adequate tongue mobility and, usually, no substantial effect on taste sensation ${ }^{126-128}$. Surgery has been reported to provide good outcomes in children who are operated on at a wide variety of ages but mainly in those who are operated on before $2-3$ years ${ }^{125,127}$.

To facilitate objective assessments and accrual of accurate long-term prognostic data, surgery should, whenever possible, be restricted to a small number of units that can offer a multidisciplinary service (including an experienced surgical team) and long-term follow-up (TABLE 4, R35-38).

\section{Management of exomphalos}

Exomphalos is a cardinal feature of BWSp (BOX 2) and is preferentially associated with molecular defects occurring within the centromeric domain (IC2 LOM or CDKN1C mutations) $)^{11,14,17}$. To date, no specific recommendations have been given regarding the management of exomphalos occurring in patients with BWSp compared with isolated exomphalos in accordance with usual local practices (TABLE 4, R39).

Molecular investigations of apparently isolated exomphalos in neonates rarely detect a molecular abnormality in the absence of additional BWSp features ${ }^{132}$.

\section{Management of hypoglycaemia}

Hypoglycaemia in BWSp is due to excess insulin and occurs in $30-60 \%$ of children with BWSp $\mathrm{p}^{11,14,17}$. Although BWSp-related neonatal hypoglycaemia is often transient and resolves within a few days, in up to $20 \%$ of neonates, it can persist beyond the first week of life and require medical treatments or even pancreatectomy in the most severe cases ${ }^{20}$.

Congenital hyperinsulinism is a rare condition with a range of causes ${ }^{20,133}$. In a cohort of 501 patients with hyperinsulinism (excluding patients with focal hyperinsulinism), $\sim 6 \%$ had features of BWSp (most of whom had segmental upd(11)pat), and half of these patients underwent surgery owing to persistent hypoglycaemia after optimal medication ${ }^{27}$.

Although low plasma glucose concentrations are common during the first 24 hours of life in all neonates, by day 3, plasma glucose concentrations in neonates are similar to those of older children, with a normal range of $3.5-5.5 \mathrm{mmol} / \mathrm{l}(60-100 \mathrm{mg} / \mathrm{dl})^{134}$. A diagnosis of hyperinsulinism is based on evidence of increased insulin secretion and/or actions at the time of hypoglycaemia, including a detectable insulin level, suppressed levels of plasma $\beta$-hydroxybutyrate (ketones), suppressed levels of plasma free fatty acids and a glycaemic response to glucagon. Diagnosis should be made in consultation with an endocrinologist who is familiar with hyperinsulinism.

Neonates with suspected BWSp should be screened for hypoglycaemia (TABLE 4, R40,41) before discharge from the nursery. Neonates with confirmed hypoglycaemia should be treated to maintain a plasma glucose concentration $>3.9 \mathrm{mmol} / \mathrm{l}(>70 \mathrm{mg} / \mathrm{dl})^{134}$. Management of hyperinsulinism includes medical therapies such as diazoxide and somatostatin analogues (such as octreotide and lanreotide). Surgery (pancreatectomy) might be indicated if persistent hypoglycaemia occurs despite maximal medical therapies ${ }^{135}$. New therapies such as mechanistic target of rapamycin (mTOR) inhibitors (sirolimus) or glucagon-like peptide 1 receptor (GLP1R) antagonists have been used in the treatment of hyperinsulinism and very recently in $\mathrm{BWSp}^{136}$; however, to date, no specific management (medical or surgical) for hyperinsulinism has been evaluated in the context of BWSp (TABLE 4, R42).

Two genes that are implicated in congenital hyperinsulinism, $A B C C 8$ and $K C N J 11$, map to chromosome $11 \mathrm{p}$, and some patients with BWSp might carry a heterozygous mutation in either gene ${ }^{27}$, although these are rare. If these genes are included in the isodisomy, then homozygosity for the mutation in disomic cells produces severe hypoglycaemia ${ }^{27}$. In cases of hypoglycaemia with hyperinsulinism and without other traits suggestive of BWSp, investigations for 11p15.5 methylation abnormalities might be considered.

\section{Management of cardiac lesions}

Congenital heart disease is more prevalent in BWS than in the general paediatric population, and cardiac defects occur in up to $13-20 \%$ of patients with BWS ${ }^{12,17,23}$ (TABLE 4, R44). Minor anatomical defects (for example, cardiomegaly, patent ductus arteriosus or patent foramen ovale and interatrial or interventricular defects) require echocardiographic monitoring until usual spontaneous resolution occurs (TABLE 4, R45). More severe defects might require surgical correction, although the management will be similar to that in sporadic cases of heart disease (TABLE 4, R47).

Congenital long QT syndrome has been reported in two families with BWS harbouring an intragenic deletion and a translocation at IC2 leading to inactivation of the KCNQ1 gene, which, although very rare, is associated with a risk of sudden death ${ }^{85,102}$ (TABLE 4, R46).

\section{Management of neurological features}

Cognitive development is usually normal in patients with BWSp; however, developmental delay can be associated with prematurity, severe hypoglycaemia, unbalanced chromosome rearrangements or paternal genome-wide $\mathrm{UPD}^{137}$ (TABLE 4, R48). The differential diagnosis should be carefully considered in patients with presumptive BWS and learning disability and without an 11p15 anomaly, as some overgrowth disorders (for example, Sotos syndrome, Malan syndrome and Simpson-Golabi-Behmel syndrome) are more frequently associated with developmental delay ${ }^{30,138-144}$ (TABLE 4, R49; see Supplementary information S3 (table)). 
Malformations of the central nervous system (for example, abnormal posterior fossae (including DandyWalker malformations) or abnormal corpus callosum or septum pellucidum) have been reported in rare patients with BWSp (chiefly with a defect involving IC2) ${ }^{83,145}$, and these features might need to be considered in children with neurological symptoms or signs (TABLE 4, R50).

\section{Management of renal complications}

The prevalence of nephro-urological anomalies in BWSp is $28-61 \%{ }^{146}$. A variety of anomalies have been described; cortical and medullary cysts occur in $~ 10 \%$ of patients with BWSp and the prevalence of hypercalciuria and nephrolithiasis is increased compared with that in the general population ${ }^{147}$. Although not all nephrourological anomalies detected by ultrasonography will be of clinical importance, a minority of anomalies might be severe (and usually detectable prenatally) and require medical or surgical management. Severe vesicoureteral reflux might cause kidney damage and recurrent urinary tract infections ${ }^{148}$. In addition, nephromegaly might be a marker of increased risk of Wilms tumour ${ }^{146}$.

Renal anomalies might occur in all molecular subtypes of BWSp, but only certain groups might be offered regular renal imaging for tumour surveillance. Management of the nephro-urological aspects of BWSp should be pragmatic and balance the benefits of presymptomatic diagnosis and treatment of critical obstructions and urinary tract infections for preserving renal function with the drawbacks of over-investigation for benign variants detected by surveillance. Thus, we recommend a nephro-urological evaluation at clinical diagnosis and at the time of adult transition for any patient with BWSp and screening for nephrocalcinosis and/or stones in only patients who undergo abdominal USS for tumour screening (TABLE 4, R51-55).

\section{BWSp and embryonal tumours}

Embryonal tumours occur in $\sim 8 \%$ of children with BWS ${ }^{149}$. The most common types of embryonal tumours are Wilms tumour (52\% of all tumours), hepatoblastoma ( $14 \%$ of all tumours), neuroblastoma ( $10 \%$ of all tumours), rhabdomyosarcoma (5\% of all tumours) and adrenal carcinoma ( $3 \%$ of all tumours) ${ }^{14}$. Although there are some differences in mean age at diagnosis between tumour types, the overall cancer risk is highest in the first 2 years of life, and clinical experience suggests that the cancer risk then declines progressively before puberty, approaching the cancer risk of the general population. Currently, there is no evidence of an increased risk of malignant tumours in adulthood (Supplementary information S4 (table)).

The tumour risk correlates with the BWSp molecular subgroup; patients with segmental upd(11)pat and IC1 GOM have a higher tumour risk than patients with CDKN1C mutations and IC2 $\mathrm{LOM}^{58}$. The four main molecular subgroups are characterized by a cancer risk gradient, with the highest risk in cases of IC1 GOM (28\% risk), followed by segmental upd(11)pat (16\% risk), CDKN1C mutation (6.9\% risk) and IC2 LOM $(2.6 \% \text { risk })^{14}$. In addition, there are also differences in the tumour types observed between molecular subgroups. Patients with IC1 GOM are mostly predisposed to developing Wilms tumour (observed in $24 \%$ of patients and accounting for $95 \%$ of malignancies in this group $)^{14,15,149}$. Conversely, patients with IC2 LOM and CDKN1C mutations do not usually develop Wilms tumour but rather develop other tumours such as hepatoblastoma, rhabdomyosarcoma and neuroblastoma. Thus, a study from 2016 reported a prevalence of Wilms tumour of $\sim 0.2 \%$ (2/995) in patients BWSp and IC2 LOM ${ }^{149}$; although a report from 2017 suggested that the risk of Wilms tumour in patients with IC2 LOM is underestimated, only a single patient with Wilms tumour and an IC2 epimutation was observed ${ }^{150}$. Therefore, when put together with previous reports of Wilms tumour in large cohorts of patients with BWSp, the overall prevalence of Wilms tumour with IC2 LOM is probably well below $1 \%{ }^{151}$. Patients with CDKN1C mutations are mostly predisposed to neuroblastoma ${ }^{14,15,149}$. Patients with segmental upd(11) pat are predisposed to develop any of the tumour types seen in BWSp (TABLE 5). Individuals with genome-wide paternal UPD seem to have a high risk of developing tumour types similar to those with segmental upd(11) pat but with an increased incidence of hepatic and/or adrenal tumours extending into adolescence and young adulthood $^{63,64,137,152}$.

Specific studies investigating the tumour risk in patients with isolated lateralized overgrowth and clinically diagnosed BWS with negative molecular testing are lacking. It seems plausible that the cancer risk in patients with isolated lateralized overgrowth who fall within the BWSp is linked to the type of $11 \mathrm{p} 15.5$ molecular anomaly. Indeed, the tumour risk in patients with isolated lateralized overgrowth and segmental upd(11) pat is estimated to be as high as $32-50 \%^{121,153}$.

\section{Tumour surveillance strategies}

Tumour screening in patients with inherited cancer predisposition syndromes aims to improve patient survival and reduce morbidity through earlier detection of tumours. However, no surveillance protocol can detect every tumour, and there are both benefits and drawbacks to screening - the latter include the financial costs, morbidity that can result from investigating asymptomatic benign lesions detected on surveillance and psychosocial burden of repeated investigations for the patient and family. There is no generally accepted risk threshold for instigating tumour screening strategies, and it might vary according to regional medical and medicolegal practices and local health-care systems. Although screening is considered for a tumour risk $>1 \%$ in the USA, a risk of $5 \%$ might be considered an appropriate threshold in Europe $\mathrm{e}^{10,154}$. Various protocols have been suggested for tumour surveillance in BWSp, usually comprising abdominal USS with or without measurement of AFP levels at various ages and intervals during infancy ${ }^{14,15,155}$. Traditionally, although most protocols have been applied to all cases of BWSp, the definition of specific epigenotype-tumour risk correlations provides a basis for more targeted surveillance protocols. 
Table 5 | Proposed tumour surveillance protocol for Beckwith-Wiedemann spectrum

\begin{tabular}{|c|c|c|c|}
\hline Tumour risk (\% of patients)* & Tumour type for surveillance & Surveillance procedures & Timing \\
\hline \multicolumn{4}{|l|}{ IC2 LOM } \\
\hline $\begin{array}{l}\text { - Overall risk (2.6\%) } \\
\text { - Hepatoblastoma }(0.7 \%) \\
\text { - Rhabdomyosarcoma }(0.5 \%) \\
\text { - Neuroblastoma }(0.5 \%) \\
\text { - Thyroid cancer }(0.3 \%) \\
\text { - Wilms tumour }(0.2 \%) \\
\text { - Melanoma }(0.1 \%)\end{array}$ & $\begin{array}{l}\text { Tumour incidence lower than other } \\
\text { molecular subgroups; extremely } \\
\text { variable tumour spectrum; only half } \\
\text { of tumours arise in the abdomen }\end{array}$ & $\begin{array}{l}\text { - No routine USS surveillance } \\
\text { - Clinical assessment and USS } \\
\text { in response to signs and/or } \\
\text { symptoms or parental concerns }\end{array}$ & - \\
\hline $\begin{array}{l}\text { - Overall risk (28.1\%) } \\
\text { - Wilms tumour (24\%) } \\
\text { - Neuroblastoma (0.7\%) } \\
\text { - Pancreatoblastoma (0.7\%) }\end{array}$ & Wilms tumour & Abdominal USS & $\begin{array}{l}\text { Every } 3 \text { months from } \\
\text { diagnosis until age } 7 \text { years }\end{array}$ \\
\hline \multicolumn{4}{|l|}{ upd(11)pat } \\
\hline \multicolumn{4}{|l|}{ CDKN1C mutation } \\
\hline $\begin{array}{l}\text { - Overall risk (6.9\%) } \\
\text { - Wilms tumour (1.4\%) } \\
\text { - Neuroblastoma (4.2\%) } \\
\text { - Acute lymphoblastic leukaemia (1.4\%) }\end{array}$ & Neuroblastoma & Abdominal USS & $\begin{array}{l}\text { Every } 3 \text { months from } \\
\text { diagnosis until age } 7 \text { years }\end{array}$ \\
\hline \multicolumn{4}{|c|}{ Classical BWS with negative molecular tests } \\
\hline
\end{tabular}

Screening for Wilms tumour. Abdominal USS is the preferred modality for Wilms tumour screening. The doubling time of Wilms tumour cells has been estimated to be 11-13 days ${ }^{156}$, and USS is recommended every 3-4 months ${ }^{157,158}$. Given the high survival of individuals with Wilms tumour (90\% overall survival at 4 years), early detection of Wilms tumour by surveillance is predicted to only marginally impact survival; however, diagnosis at an earlier stage might reduce the burden of treatment-related morbidity ${ }^{159-162}$.

If Wilms tumour screening is targeted by BWSp molecular subgroup, patients with IC1 GOM and segmental upd(11)pat are at the highest risk, and several groups have suggested that patients with IC2 LOM should not be screened using USS in order to avoid excessive medicalization and possible false-positive results $^{149,154}$
Screening for hepatoblastoma. The risk of hepatoblastoma in patients with BWS is $>2,000$-fold higher than in the general population, and hepatoblastoma is the second most common tumour type in BWS ${ }^{21}$. However, specific studies evaluating hepatoblastoma screening in BWSp are lacking. Abdominal USS is a first-line investigation in children with a suspected liver mass, although not all parts of the liver can be imaged easily and small tumours might be missed ${ }^{159}$. Concerns about the sensitivity of abdominal USS led to suggestions that it should be combined with measurements of serum levels of AFP, which is secreted by $>95 \%$ of hepatoblastomas ${ }^{163-165}$. Treatment and outcome of patients with hepatoblastoma are closely connected to tumour stage at diagnosis, and preliminary data suggested that patients with BWSp and hepatoblastoma who are screened for AFP have an earlier stage at diagnosis and a better 
prognosis than unscreened patients and that increased serum AFP levels might precede hepatoblastoma detection by USS ${ }^{166}$. However, this hypothesis is unproved, and further data are required. In the paediatric setting, interpreting serum AFP levels can be complex owing to the wide range and variable concentrations in early infancy ${ }^{167,168}$. Serum AFP levels might be higher in babies with BWSp and without hepatoblastoma than in agematched healthy controls ${ }^{169}$. In view of the burden of repeated venepuncture and the complexity of interpreting elevated AFP levels, it has been debated whether the benefits of AFP screening in BWSp outweigh the drawbacks ${ }^{170-173}$, and the consensus voted not to recommend AFP screening (TABLE 4, R64).

Screening for neuroblastoma. Although reported in all BWSp molecular subgroups, neuroblastomas are preferentially associated with CDKN1C mutations, with a frequency of $\sim 4 \%{ }^{14}$ (TABLE 5). Detection of asymptomatic neuroblastomas by determination of the urinary tumour markers vanillylmandelic acid and homovanillic acid and/or the catecholamine to creatinine ratio combined with three monthly USSs until age $2-3$ years has been suggested ${ }^{149}$. However, previous neuroblastoma screening strategies using urinary markers in largescale paediatric settings had a very minor influence on the related morbidity and mortality ${ }^{174,175}$, and there is currently no evidence that neuroblastoma screening in BWSp improves treatment and survival (TABLE 4, R65).

Surveillance for other tumour types. Screening for adrenal carcinoma can be undertaken using clinical evaluation, adrenal USS and determination of serum dehydroepiandrosterone sulfate concentrations every 4-6 months ${ }^{176}$. However, adrenal carcinoma is rare in BWS (even in patients with genome-wide paternal UPD, who are at the highest risk), and there is no data on the utility of such screening strategies in BWSp.

\section{Consensus tumour surveillance protocol}

The consensus group agreed that tumour surveillance should be targeted to those molecular subgroups of BWSp that are at highest risk and that children with BWSp and IC2 LOM should not be offered routine USS (TABLE 4, R60) (although, there should be a low threshold for investigation in response to symptoms or parental concern). Other BWSp molecular subgroups and patients with classical BWS and no detectable molecular anomaly should be offered abdominal USS every 3 months until age 7 years (TABLE 4, R57-59,61-63; TABLE 5). It was agreed that AFP measurements should not be offered routinely because the incidence of hepatoblastoma was judged too low to warrant specific screening, the influence of surveillance of patients and families is unclear, and the difficulties in interpretation might lead to false-positive results (TABLE 4, R64). Nevertheless, in specific health-care systems, clinicians might currently vary from the proposed protocol, especially when regional protocols are available, pending the outcome of the results of prospective studies of targeted and universal surveillance.
The consensus surveillance protocol enables $\sim 50 \%$ of children with BWSp at low tumour risk to be spared 3 monthly USSs, and although the risk of Wilms tumour, for example, is small in patients with a CDKN1C mutation, applying a common surveillance modality (abdominal USS rather than renal USS in some subgroups, liver USS in other subgroups and so on) in all the groups to be screened avoids the potential for confusion with more complicated regimens. It should be noted that the agreed protocol differs from that recommended recently by the American Association for Cancer Research (AACR) Childhood Cancer Predisposition Workshop, which adopted a 1\% risk threshold for surveillance and therefore recommended abdominal USS and AFP screening for all cases of BWSp ${ }^{155}$. Both the AACR group and this consensus group made these decisions on the basis of similar data for tumour risks in different molecular subgroups but came to differing conclusions with regard to adopting a targeted screening approach. It should be noted that the AACR group consisted predominantly of experts from North America, whereas the international BWS consensus group was composed predominantly of experts from European centres, where targeted screening has already been adopted in some countries ${ }^{14,154}$. Thus, the difference in screening recommendations between the two groups reflects mainly the different medical and medicolegal cultures in North America and Europe. Although a universally agreed upon screening protocol would usually be preferable, taking into account the different conclusions between the experts of both the AACR group and this consensus group, it is reasonable that at this time, screening protocols could be different between Europe and North America. Such diversity of practice can be helpful, as careful audit of the results of the two protocols can help further refine our recommendations at future international consensus meetings ${ }^{155}$.

\section{Management of BWSp-related tumours}

Children with BWSp and Wilms tumour, when compared with non-syndromic children with Wilms tumour, present with less metastatic disease because of earlier stage disease, fewer anaplastic tumours and a higher incidence of bilateral synchronous or metachronous recurrence ${ }^{161}$ (TABLE 4, R67). The latter seem to be connected to the presence of multifocal or diffuse nephrogenic rests in one or both kidneys (nephroblastomatosis) ${ }^{177}$, a feature that is not easily distinguishable from Wilms tumour on standard imaging ${ }^{160}$. Although patients with BWSp and Wilms tumour who are diagnosed using abdominal USS surveillance have a smaller tumour size than children with sporadic Wilms tumour ${ }^{157}$, overall survival values are similar (at least $90 \%$ at 4 years) ${ }^{161}$. Smaller Wilms tumours are more amenable to partial nephrectomy and nephron-sparing strategies (such as partial nephrectomy) are particularly preferred in patients with BWSp given the potential co-occurrence of progressive non-malignant renal diseases and bilateral Wilms tumours ${ }^{158}$. Data from 2016 show comparable outcomes after nephron-sparing surgery and total nephrectomy in patients with BWSp and Wilms tumour ${ }^{160}$. 


\section{Late-onset complications}

Features of BWSp such as macroglossia and postnatal overgrowth tend to ameliorate with age, and therefore, BWSp is often likely overlooked in adults unless there is a prior diagnosis in childhood. There is a paucity of information on long-term outcomes and late-onset complications in adults with BWSp (TABLE 4, R70). Concerns about potential adult-onset complications that are not directly related to childhood features of BWSp fall into four areas.

Neoplasia. Despite the link with embryonal neoplasia, there is no apparent association between BWSp and predisposition to common adult-onset carcinomas. Although rare endocrine tumours have been reported in adults with BWSp ${ }^{62,152,178-180}$ (Supplementary information S4 (table)), there is no evidence of a specific tumour risk that might justify surveillance. However, follow-up of large series of adults with BWS has not been performed. Children with BWSp who are treated for embryonal tumours might develop late-onset complications from surgery, radiotherapy or chemotherapy, similar to children with sporadic tumours.

Cardiovascular defects. Patients with congenital heart disease require appropriate follow-up in adult specialty clinics. Although rare, cardiovascular defects might be diagnosed for the first time in adulthood ${ }^{181}$, but routine screening is not indicated. Patients with rare IC2 CNVs and/or rearrangements that might predispose to long QT syndrome require follow-up throughout adulthood.

Infertility. Although congenital anomalies of the urogenital tract (for example, bicornuate uterus) have been described in BWSp ${ }^{178,181}$, there is no clear evidence of excess fertility problems in women with BWSp. Reduced fecundity has been described in affected males (compared with females $)^{182}$, but the frequency of infertility in men with BWSp is unknown.

Renal anomalies. Although examples of the diagnosis of renal anomalies in adults with BWSp have been reported ${ }^{178}$, it is assumed that if renal USS was performed, renal abnormalities would usually be detected in childhood.

Although regular surveillance (for example, echocardiography, renal function testing and evaluation of hearing) has been suggested for adults with BWSp ${ }^{181}$, in the absence of abnormalities detected during childhood surveillance, the detection rate of such investigations in asymptomatic adults with BWSp is likely to be low and could pose problems with health insurance. The consensus group agreed that a detailed clinical review and renal USS (Supplementary information S5 (table)) should be undertaken at age 16 years, and specific recommendations for continued surveillance based on only ongoing problems were agreed upon (TABLE 4, R68). Adults with BWSp should be encouraged to seek genetic counselling advice before starting a family (TABLE 4, R69). At that stage, any potential concerns about fertility can be reviewed and referrals for further investigation made as appropriate.

\section{Psychological and counselling aspects}

The diagnosis of a disorder such as BWSp can have wide-ranging effects on the psychological and social well-being of families. Although the precise effects will vary between families and will be influenced by individual medical and social factors, and considering that each family might face different challenges, it is important that all health-care professionals are aware of the wider non-medical issues that might be relevant to the family (TABLE 4, R71). Information on the psychosocial aspects that might be specific to BWSp is sparse. In many cases, there is no previous relevant family history and the parents are not prepared for the diagnosis. Issues such as tumour risk can be worrying, and it is apparent that differing medical practices and recommendations might cause parental uncertainty and anxiety. A survey of parents of children with BWSp who have macroglossia revealed widespread parental concerns about the 'negative cosmetic appearances' of a large protruding tongue and persistent drooling, which led to strangers staring and questioning whether their children had learning difficulties ${ }^{128}$. Parents are also concerned that this might lead to teasing by other children, and a retrospective questionnaire survey revealed an apparent increase in emotional difficulties and problems with peers in children with BWSp ${ }^{183}$. Health-care professionals should be aware that psychosocial difficulties might occur and should be prepared to refer families to specialists such as genetic counsellors, social workers and psychologists, as appropriate. As support groups can have key roles in helping families adjust to the diagnosis, share their concerns and experiences and obtain the correct care and support, all families should be given the contact details of relevant groups (TABLE 4, R72).

\section{Conclusions}

The recommendations of the first international BWS consensus group described in this Consensus Statement provide a framework for improving the diagnosis and management of BWSp. As BWSp is characterized by complex genetics and variable multisystem phenotypes, it is important that a lead clinician is identified for each patient (TABLE 4, R22) to ensure coordination of the numerous aspects of care throughout childhood (Supplementary information S5 (table)). The proposed diagnostic and care pathways are intended to be practical and cost-effective (for example, targeting tumour surveillance to high-risk groups should reduce costs compared with universal surveillance strategies). Nevertheless, in some health-care systems and medicolegal environments, further evidence might be required to shift clinical practice (for example, tumour surveillance in North America). Thus, it is important that implementation of these consensus recommendations be accompanied by prospective audits in order to expand the evidence base for future consensus initiatives. 
1. Eggermann, T. et al. Imprinting disorders: a group of congenital disorders with overlapping patterns of molecular changes affecting imprinted loci. Clin Epigenet. 7, 123 (2015)

2. Choufani, S., Shuman, C. \& Weksberg, R. Beckwith Wiedemann syndrome. Am. J. Med. Genet. Part C Semin. Med. Genet. 154C, 343-354 (2010)

3. Mussa, A. et al. Prevalence of beckwith-wiedemann syndrome in North West of Italy. Am. J. Med. Genet Part A 161, 2481-2486 (2013).

4. de Villiers, M. R., de Villiers, P. J. T. \& Kent, A. P. The Delphi technique in health sciences education research. Med. Teach. 27, 639-643 (2005).

5. Beckwith, J. B. Extreme cytomegaly of the adrenal fetal cortex, omphalocele, hyperplasia of kidneys and pancreas, and Leydig-cell hyperplasia: another syndrome in Annual Meeting of Western Society of Pediatric Research (Los Angeles, California, 1963).

6. Wiedemann, H. R. Familial malformation complex with umbilical hernia and macroglossia - a 'new syndrome'? J. Genet. Hum. 13, 223-232 (1964).

7. Henry, I. et al. Uniparental paternal disomy in a genetic cancer-predisposing syndrome. Nature 351 , 665-667 (1991).

8. Reik, W. et al. Imprinting mutations in the Beckwith Wiedemann syndrome suggested by altered imprinting pattern in the IGF2-H19 domain Hum. Mol. Genet. 4, 2379-2385 (1995).

9. Hatada, I. et al. An imprinted gene p57KIP2 is mutated in Beckwith-Wiedemann syndrome. Nat. Genet. 14, 171-173 (1996).

10. Kalish, J. M. et al. Nomenclature and definition in asymmetric regional body overgrowth. Am. J. Med Genet. Part A 173, 1735-1738 (2017).

11. Ibrahim, A. et al. Methylation analysis and diagnostics of Beckwith-Wiedemann syndrome in 1,000 subjects. Clin. Epigenet. 6, 11 (2014).

12. Elliott, M. et al. Clinical features and natural history of Beckwith-Wiedemann syndrome: presentation of 74 new cases. Clin. Genet. 46, 168-174 (1994).

13. Weksberg, R., Shuman, C. \& Beckwith, J. B. Beckwith Wiedemann syndrome. Eur. J. Hum. Genet. 18, 8-14 (2010).

14. Maas, S. M. et al. Phenotype, cancer risk, and surveillance in Beckwith-Wiedemann syndrome depending on molecular genetic subgroups. Am. J. Med. Genet. A 170A, 2248-2260 (2016).

15. Brioude, F. et al. Beckwith-wiedemann syndrome: growth pattern and tumor risk according to molecular mechanism, and guidelines for tumor surveillance. Horm. Res. Paediatr. 80, 457-465 (2014).

16. Mussa, A., Russo, S., Larizza, L., Riccio, A. \& Ferrero, G. B. (Epi)genotype-phenotype correlations in Beckwith-Wiedemann syndrome: a paradigm for genomic medicine. Clin. Genet. 89, 403-415 (2016).

17. Mussa, A. et al. (Epi)genotype-phenotype correlations in Beckwith-Wiedemann syndrome. Eur. J. Hum. Genet. 24, 183-190 (2016).

18. Gaston, V. et al. Analysis of the methylation status of the KCNQ1OT and H19 genes in leukocyte DNA for the diagnosis and prognosis of Beckwith-Wiedemann syndrome. Eur. J. Hum. Genet. 9, 409-418 (2001).

19. Sotelo-Avila, C., Gonzalez-Crussi, F. \& Fowler, J. W. Complete and incomplete forms of BeckwithWiedemann syndrome: their oncogenic potential. J. Pediatr. 96, 47-50 (1980).

20. Munns, C. F. \& Batch, J. A. Hyperinsulinism and Beckwith-Wiedemann syndrome. Arch. Dis. Child Fetal Neonatal Ed. 84, F67-69 (2001).

21. DeBaun, M. R. \& Tucker, M. A. Risk of cancer during the first four years of life in children from The Beckwith-Wiedemann Syndrome Registry. J. Pediatr. 132, 398-400 (1998).

22. Zarate, Y. a et al. Experience with hemihyperplasia and Beckwith-Wiedemann syndrome surveillance protocol. Am. J. Med. Genet. A 149A, 1691-1697 (2009).

23. Pettenati, M. et al. Wiedemann-Beckwith syndrome: presentation of clinical and cytogenetic data on 22 new cases and review of the literature. Hum. Genet 74, 143-154 (1986)

24. DeBaun, M. et al. Epigenetic alterations of $\mathrm{H} 19$ and LIT 1 distinguish patients with Beckwith-Wiedemann syndrome with cancer and birth defects. Am. J. Hum. Genet. 70, 604-611 (2002)

25. Mussa, A. et al. Nephrological findings and genotypephenotype correlation in Beckwith-Wiedemann syndrome. Pediatr. Nephrol. 27, 397-406 (2012).

26. Lin, H. Y. Epigenotype, genotype, and phenotype analysis of patients in Taiwan with BeckwithWiedemann syndrome. Mol. Genet. Metab. 119, 8-13 (2016).
27. Kalish, J. et al. Congenital hyperinsulinism in children with paternal $11 \mathrm{p}$ uniparental isodisomy and Beckwith-Wiedemann syndrome. J. Genet. 53, 53-61 (2016).

28. Hussain, K. et al. Hyperinsulinemic hypoglycaemia in Beckwith-Wiedemann syndrome due to defects in the function of pancreatic beta-cell adenosine triphosphate-sensitive potassium channels. J. Clin Endocrinol. Metab. 90, 4376-4382 (2005).

29. Viljoen, D. \& Ramesar, R. Evidence for paternal imprinting in familial Beckwith-Wiedemann syndrome. J. Med. Genet. 29, 221-225 (1992)

30. Li, M. et al. GPC3 mutation analysis in a spectrum of patients with overgrowth expands the phenotype of Simpson-Golabi-Behmel syndrome. Am. J. Med. Genet. 102, 161-168 (2001)

31. Halliday, J., Oke, K., Breheny, S., Algar, E. \& Amor, D. J. Beckwith-Wiedemann syndrome and IVF: a case-control study. Am. J. Hum. Genet. 75, 526-528 (2004).

32. Cox, G. et al. Intracytoplasmic sperm injection may increase the risk of imprinting defects. Am. J. Hum. Genet. Genet. A 71, 162-164 (2002).

33. Ørstavik, K. H. et al. Another case of imprinting defect in a girl with Angelman syndrome who was conceived by intracytoplasmic semen injection. Am. J. Hum. Genet. 72, 218-219 (2003).

34. Gicquel, C. et al. In vitro fertilization may increase the risk of Beckwith-Wiedemann syndrome related to the abnormal imprinting of the KCNQ1OT gene. Am. J. Hum Genet. 15, 1106-1107 (2003)

35. DeBaun, M. R., Niemitz, E. L. \& Feinberg, A. P. Association of in vitro fertilization with Beckwith Wiedemann syndrome and epigenetic alterations of LIT1 and H19. Am. J. Hum. Genet. 72, 156-160 (2003)

36. Maher, E. R. et al. Beckwith-Wiedemann syndrome and assisted reproduction technology (ART). J. Med Genet. 40, 62-64 (2003).

37. Mussa, A. et al. Assisted reproductive techniques and risk of Beckwith-Wiedemann syndrome. Pediatrics 140 e20164311 (2017).

38. Källén, B., Finnström, O., Nygren, K. \& Olausson, P. In vitro fertilization (IVF) in Sweden: risk for congenital malformations after different IVF methods. Birth Defects Res. A Clin. Mol. Teratol 73, 162-169 (2005).

39. Lidegaard, O., Pinborg, A. \& Andersen, A. Imprinting diseases and IVF: Danish National IVF cohort study. Hum. Reprod. 20, 950-954 (2005).

40. Lim, D. et al. Clinical and molecular genetic features of Beckwith-Wiedemann syndrome associated with assisted reproductive technologies. Hum. Reprod. 24 741-747 (2009)

41. Horsthemke, B. \& Ludwig, M. Assisted reproduction: the epigenetic perspective. Hum. Reprod. Updat. 11 473-482 (2005)

42. Doornbos, M. E., Maas, S. M., McDonnell, J., Vermeiden, J. P. W. \& Hennekam, R. C. M. Infertility, assisted reproduction technologies and imprinting disturbances: a Dutch study. Hum. Reprod. 22 2476-2480 (2007)

43. Khoueiry, R. et al. Dynamic $\mathrm{CpG}$ methylation of the KCNQ1OT1 gene during maturation of human oocytes. J. Med. Genet. 45, 583-588 (2008)

44. Fauque, P. et al. In vitro fertilization and embryo culture strongly impact the placental transcriptome in the mouse model. PLOS One 5, e9218 (2010).

45. Doherty, A. S., Mann, M. R., Tremblay, K. D., Bartolomei, M. S. \& Schultz, R. M. Differential effects of culture on imprinted $\mathrm{H} 19$ expression in the preimplantation mouse embryo. Biol. Reprod. 62 , 1526-1535 (2000)

46. Chen, Z., Robbins, K. M., Wells, K. D. \& Rivera, R. M Large offspring syndrome. Epigenetics 8, 591-601 (2013)

47. Monk, D. et al. Recommendations for a nomenclature system for reporting methylation aberrations in imprinted domains. Epigenetics http://dx.doi.org/10.1 080/15592294.2016.1264561 (2016).

48. Eggermann, T. et al. Clinical utility gene card for Beckwith-Wiedemann Syndrome. Eur. J. Hum. Genet. 22, 7-10 (2013)

49. Weksberg, R. et al. Discordant KCNQ1 OT imprinting in sets of monozygotic twins discordant for Beckwith-Wiedemann syndrome. Hum. Mol. Genet. 11, 1317-1325 (2002)

50. Bliek, J. et al. Lessons from BWS twins: complex maternal and paternal hypomethylation and a common source of haematopoietic stem cells. Eur. J. Hum. Genet. 17, 1625-1634 (2009).
51. Eggermann, K. et al. EMON best practice guidelines for the molecular genetic testing and reporting of chromosome 11 p 15 imprinting disorders - SilverRussell and Beckwith-Wiedemann syndrome. Eur. J. Hum. Genet. 24, 1377-1387 (2016).

52. Priolo, M. et al. MS-MLPA is a specific and sensitive technique for detecting all chromosome 11 p 15.5 imprinting defects of BWS and SRS in a single-tube experiment. Eur. J. Hum. Genet. 16, 565-571 (2008).

53. Scott, R. H. et al. Methylation-specific multiplex ligation-dependent probe amplification (MS-MLPA) robustly detects and distinguishes $11 \mathrm{p} 15$ abnormalities associated with overgrowth and growth retardation. J. Med. Genet. 45, 106-113 (2008).

54. Russo, S. et al. A multi-method approach to the molecular diagnosis of overt and borderline 11 p 15.5 defects underlying Silver-Russell and BeckwithWiedemann syndromes. Clin. Epigenet. 8, 23 (2016).

55. Baskin, B. et al. High frequency of copy number variations (CNVs) in the chromosome $11 \mathrm{p} 15$ region in patients with Beckwith-Wiedemann syndrome. Hum. Genet. 133, 321-330 (2014)

56. Liu, W. et al. Rapid diagnosis of imprinting disorders involving copy number variation and uniparental disomy using genome-wide SNP microarrays. Cytogenet. Genome Res. 146, 9-18 (2015).

57. Cardarelli, L. et al. Silver-Russell syndrome and Beckwith-Wiedemann syndrome phenotypes associated with $11 \mathrm{p}$ duplication in a single family. Pediatr. Dev. Pathol. 13, 326-330 (2010)

58. Cooper, W. N. et al. Molecular subtypes and phenotypic expression of Beckwith-Wiedemann syndrome Eur. J. Hum. Genet. 13, 1025-1032 (2005).

59. Keren, B. et al. SNP arrays in beckwith-wiedemann syndrome: an improved diagnostic strategy. Eur. J. Med. Genet. 56, 546-550 (2013).

60. Lukova, M., Todorova, A., Todorov, T. \& Mitev, V. Different methylation patterns in BWS/SRS cases clarified by MS-MLPA. Mol. Biol. Rep. 40, 263-268 (2012).

61. Romanelli, V. et al. Beckwith-Wiedemann syndrome and uniparental disomy $11 \mathrm{p}$ : fine mapping of the recombination breakpoints and evaluation of several techniques. Eur. J. Hum. Genet. 19, 416-421 (2011).

62. Romanelli, V. et al. Constitutional mosaic genomewide uniparental disomy due to diploidisation: an unusual cancer-predisposing mechanism. J. Med Genet. 48, 212-216 (2011)

63. Gogiel, M. et al. Genome-wide paternal uniparental disomy mosaicism in a woman with BeckwithWiedemann syndrome and ovarian steroid cell tumour. Eur. J. Hum. Genet. 21, 788-791 (2013).

64. Wilson, M. et al. The clinical phenotype of mosaicism for genome-wide uniparental disomy: two new reports. Am. J. Med. Genet. A 146A, 137-148 (2008)

65. Inbar-Feigenberg, M. et al. Mosaicism for genomewide paternal uniparental disomy with features of multiple imprinting disorders: Diagnostic and management issues. Am. J. Med. Genet. Part A 161 , 13-20 (2013)

66. Johnson, J. P., Waterson, J., Schwanke, C. \& Schoof, J. Genome-wide androgenetic mosaicism. Clin. Genet. 85, 282-285 (2014).

67. Ohtsuka, Y et al. Identification of consensus motifs associated with mitotic recombination and clinical characteristics in patients with paternal uniparental isodisomy of chromosome 11. Hum. Mol. Genet. 25 1406-1419 (2016).

68. Sparago, A. et al. Microdeletions in the human $\mathrm{H} 19$ DMR result in loss of IGF2 imprinting and BeckwithWiedemann syndrome. Nat. Genet. 36, 958-960 (2004).

69. Prawitt, D. et al. Microdeletion of target sites for insulator protein CTCF in a chromosome $11 \mathrm{p} 15$ imprinting center in Beckwith-Wiedemann syndrome and Wilms' tumor. Proc. Natl Acad. Sci. USA 102 4085-4090 (2005).

70. Sparago, A. et al. Mechanisms causing imprinting defects in familial Beckwith-Wiedemann syndrome with Wilms' tumour. Hum. Mol. Genet. 16, 254-264 (2007).

71. Demars, J. et al. Analysis of the IGF2/H19 imprinting control region uncovers new genetic defects, including mutations of OCT-binding sequences, in patients with 11 p 15 fetal growth disorders. Hum. Mol. Genet. 19. 803-814 (2010)

72. Poole, R. L. et al. Beckwith-Wiedemann syndrome caused by maternally inherited mutation of an OCT binding motif in the IGF2/H19-imprinting control region, ICR1. Eur. J. Hum. Genet. 20, 240-243 (2012). 
73. Abi Habib, W. et al. Extensive investigation of the IGF2/H19 imprinting control region reveals nove OCT 4/SOX2 binding site defects associated with specific methylation patterns in Beckwith-Wiedemann syndrome. Hum. Mol. Genet. 23, 5763-5773 (2014).

74. Higashimoto, K. et al. A novel de novo point mutation of the OCT-binding site in the IGF2/H19-imprinting control region in a Beckwith-Wiedemann syndrome patient. Clin. Genet. 86, 539-544 (2014).

75. Rossignol, S. et al. The epigenetic imprinting defect of patients with Beckwith-Wiedemann syndrome born after assisted reproductive technology is not restricted to the 11 p15 region. J. Med. Genet. 43, 902-907 (2006).

76. Azzi, S. et al. Multilocus methylation analysis in a large cohort of $11 \mathrm{p} 15$-related foetal growth disorders (Russell Silver and Beckwith Wiedemann syndromes) reveals simultaneous loss of methylation at paterna and maternal imprinted loci. Hum. Mol. Genet. 18, 4724-4733 (2009).

77. Poole, R. L et al. Targeted methylation testing of a patient cohort broadens the epigenetic and clinical description of imprinting disorders. Am. J. Med. Genet. A 161 A, 2174-2182 (2013).

78. Bliek, J. et al. Hypomethylation at multiple maternally methylated imprinted regions including PLAGL1 and GNAS loci in Beckwith-Wiedemann syndrome. Eur. J. Hum. Genet. 17, 611-619 (2009).

79. Meyer, E. et al. Germline mutation in NLRP2 (NALP2) in a familial imprinting disorder (Beckwith-Wiedeman Syndrome). PLoS Genet. 5, e1000423 (2009).

80. Docherty, L. E. et al. Mutations in NLRP5 are associated with reproductive wastage and multilocus imprinting disorders in humans. Nat. Commun. 6, 8086 (2015).

81. Alders, $M$ et al. Methylation analysis in tongue tissue of BWS patients identifies the (EPI)genetic cause in 3 patients with normal methylation levels in blood. Eur. J. Med. Genet. 57, 293-297 (2014).

82. Lam, W. W. et al. Analysis of germline CDKN1C (p57KIP2) mutations in familial and sporadic Beckwith-Wiedemann syndrome (BWS) provides a novel genotype-phenotype correlation. J. Med. Genet. 36, 518-523 (1999)

83. Brioude, F. et al. Mutations of the Imprinted CDKN1C gene as a cause of the overgrowth BeckwithWiedemann syndrome: clinical spectrum and functional characterization. Hum. Mutat. 36, 894-902 (2015).

84. Alders, M. et al. Disruption of a novel imprinted zincfinger gene, ZNF215, in Beckwith-Wiedemann syndrome. Am. J. Hum. Genet. 66, 1473-1484 (2000).

85. Kaltenbach, S. et al. Beckwith-Wiedemann syndrome and long QT syndrome due to familial-balanced translocation $\mathrm{t}(11 ; 17)(\mathrm{p} 15.5 ; \mathrm{q} 21.3)$ involving the KCNQ1 gene. Clin. Genet. 84, 78-81 (2013).

86. Mannens, $\mathrm{M}$. et al. Parental imprinting of human chromosome region 11 p15.3-pter involved in the Beckwith-Wiedemann syndrome and various human neoplasia. Eur. J. Hum. Genet. 2, 3-23 (1994).

87. Weksberg, R. et al. Molecular characterization of cytogenetic alterations associated with the BeckwithWiedemann syndrome (BWS) phenotype refines the localization and suggests the gene for BWS is imprinted. Hum. Mol. Genet. 2, 549-556 (1993).

88. Sanchez-Delgado, M. et al. Causes and consequences of multi-locus imprinting disturbances in humans. Trends Genet. 32, 444-455 (2016).

89. Court, F. et al. Genome-wide allelic methylation analysis reveals disease-specific susceptibility to multiple methylation defects in imprinting syndromes. Hum. Mutat. 34, 595-602 (2013)

90. Maeda, T. et al. Comprehensive and quantitative multilocus methylation analysis reveals the susceptibility of specific imprinted differentially methylated regions to aberrant methylation in Beckwith-Wiedemann syndrome with epimutations. Genet. Med. 16, 903-912 (2014).

91. Docherty, L. E. et al. Genome-wide DNA methylation analysis of patients with imprinting disorders identifies differentially methylated regions associated with novel candidate imprinted genes. J. Med. Genet. 51, 229-238 (2014)

92. Tee, L. et al. Epimutation profiling in Beckwith Wiedemann syndrome: relationship with assisted reproductive technology. Clin. Epigenet. 5, 23 (2013).

93. Baple, E. L. et al. An atypical case of hypomethylation at multiple imprinted loci. Eur. J. Hum. Genet. 19 360-362 (2011)

94. Slavotinek, A., Gaunt, L. \& Donnai, D. Paternally inherited duplications of $11 \mathrm{p} 15.5$ and BeckwithWiedemann syndrome. J. Med. Genet. 34, 819-826 (1997).
95. Bliek, J. et al. Phenotypic discordance upon paternal or maternal transmission of duplications of the $11 \mathrm{p} 15$ imprinted regions. Eur. J. Med. Genet. 52, 404-408 (2009).

96. Vals, M.-A. et al. Familial 1.3-Mb 11p15.5p15.4 duplication in three generations causing Silver-Russell and Beckwith-Wiedemann syndromes. Mol. Syndr. 6, 147-151 (2015)

97. Algar, E. M. et al. Paternally inherited submicroscopic duplication at 11 p15.5 implicates insulin-like growth factor II in overgrowth and Wilms' tumorigenesis. Cancer Res. 67, 2360-2365 (2007)

98. Zollino, M. et al. A case of Beckwith-Wiedemann syndrome caused by a cryptic $11 \mathrm{p} 15$ deletion encompassing the centromeric imprinted domain of the BWS locus. J. Med. Genet. 47, 429-432 (2010).

99. Niemitz, E. L. et al. Microdeletion of LIT1 in familial Beckwith-Wiedemann syndrome. Am. J. Hum. Genet. 75, 844-849 (2004).

100. Algar, E., Dagar, V., Sebaj, M. \& Pachter, N. An 11p15 imprinting centre region 2 deletion in a family with Beckwith Wiedemann syndrome provides insights into imprinting control at CDKN1C. PLOS ONE 6, e29034 (2011)

101. Chiesa, N et al. The KCNQ1OT1 imprinting control region and non-coding RNA: new properties derived from the study of Beckwith-Wiedemann syndrome and Silver-Russell syndrome cases. Hum. Mol. Genet. 21, 10-25 (2012).

102. Gurrieri, F. et al. Mild Beckwith-Wiedemann and severe long-QT syndrome due to deletion of the imprinting center 2 on chromosome 11 p. Eur. J. Hum. Genet. 21, 965-969 (2013).

103. Cerrato, F., De Crescenzo, A. \& Riccio, A. Looking for CDKN1C enhancers. Eur. J. Hum. Genet. 22, 442-443 (2014).

104. Fryssira, H. et al. A novel large deletion of the ICR 1 region including $\mathrm{H} 19$ and putative enhancer elements. BMC Med. Genet. 16, 30 (2015).

105. Beygo, J. et al. The molecular function and clinica phenotype of partial deletions of the IGF2/H19 imprinting control region depends on the spatial arrangement of the remaining CTCF-binding sites. Hum. Mol. Genet. 22, 544-557 (2013).

106. Berland, S. et al. Evidence for anticipation in Beckwith-Wiedemann syndrome. Eur. J. Hum. Genet 21, 1344-1348 (2013)

107. Eggermann, T. et al. Prenatal molecular testing for Beckwith-Wiedemann and Silver-Russell syndromes: a challenge for molecular analysis and genetic counseling. Eur. J. Hum. Genet. 24, 784-793 (2016).

108. Paganini, L. et al. Beckwith-Wiedemann syndrome prenatal diagnosis by methylation analysis in chorionic villi. Epigenetics 10, 643-649 (2015)

109. Kagan, K. O. et al. Novel fetal and maternal sonographic findings in confirmed cases of Beckwith-Wiedemann syndrome. Prenat. Diagn. 35, 394-399 (2015).

110. Jauniaux, E., Nicolaides, K. H. \& Hustin, J. Perinatal features associated with placental mesenchyma dysplasia. Placenta 18, 701-706 (1997).

111. Gocmen, R. et al. Bilateral hemorrhagic adrenal cysts in an incomplete form of Beckwith-Wiedemann syndrome: MRI and prenatal US findings. Abdom. Imag. 30, 786-789 (2005).

112. Guanciali-Franchi, P. et al. Elevated maternal serum $\alpha$-fetoprotein level in a fetus with BeckwithWiedemann syndrome in the second trimester of pregnancy. J. Prenat. Med. 6, 7-9 (2012).

113. Boyd, P. A., Bhattacharjee, A., Gould, S., Manning, N. \& Chamberlain, P Outcome of prenatally diagnosed anterior abdominal wall defects. Arch. Dis. Child. Fetal Neonatal Ed. 78, F209-F213 (1998).

114. Nayeri, U. A., West, A. B., Grossetta Nardini, H. K. Copel, J. A. \& Sfakianaki, A. K. Systematic review of sonographic findings of placental mesenchymal dysplasia and subsequent pregnancy outcome. Ultrasound Obs. Gynecol. 41, 366-374 (2013).

115. Wangler, M. F., Chang, A. S., Moley, K. H., Feinberg, A. P. \& DeBaun, M. R. Factors associated with preterm delivery in mothers of children with Beckwith-Wiedemann syndrome: a case cohort study from the BWS registry. Am. J. Med. Genet. 134A, 187-191 (2005)

116. Romanelli, V. et al. CDKN1C mutations in HELLP/ preeclamptic mothers of Beckwith-Wiedemann Syndrome (BWS) patients. Placenta 30, 551-554 (2009).

117. Aagaard-Tillery, K. M., Buchbinder, A., Boente, M. P. \& Ramin, K. D. Beckwith-Wiedemann syndrome presenting with an elevated triple screen in the second trimester of pregnancy. Fetal Diagn. Ther. 22, 18-22 (2007).
118. Mussa, A. et al. Fetal growth patterns in BeckwithWiedemann syndrome. Clin. Genet. 90, 21-27 (2016).

119. Martin, R. A., Grange, D. K., Zehnbauer, B. \& Debaun, M. R. LIT1 and H19 methylation defects in isolated hemihyperplasia. Am. J. Med. Genet. A 134A, 129-131 (2005).

120. Tenorio, J. et al. Clinical and molecular analyses of Beckwith-Wiedemann syndrome: comparison between spontaneous conception and assisted reproduction techniques. Am. J. Med. Genet. A 170A, 2740-2749 (2016).

121. Shuman, C. et al. Constitutional UPD for chromosome $11 \mathrm{p} 15$ in individuals with isolated hemihypertrophy is associated with high tumour risk and occurs following assisted reproductive technologies. Am. J. Med. Genet. A 140A, 1497-1503 (2007).

122. Ghanem, I., Karam, J. A. \& Widmann, R. F. Surgical epiphysiodesis indications and techniques: update. Curr. Opin. Pediatr. 23, 53-59 (2011).

123. Mussa, A. et al. Recommendations of the Scientific Committee of the Italian Beckwith-Wiedemann Syndrome Association on the diagnosis, management and follow-up of the syndrome. Eur. J. Med. Genet. 59 52-64 (2015).

124. Prada, C. E., Zarate, Y. A. \& Hopkin, R. J. Genetic causes of macroglossia: diagnostic approach Pediatrics 129, e431-e437 (2012)

125. Kadouch, D. J. M., Maas, S. M., Dubois, L. \& van der Horst, C. M. A. M. Surgical treatment of macroglossia in patients with Beckwith-Wiedemann syndrome: a 20-year experience and review of the literature. Int. J. Oral Maxillofac. Surg. 41, 300-308 (2012).

126. Gasparini, G., Saltarel, A., Carboni, A., Maggiulli, F. \& Becelli, R. Surgical management of macroglossia: discussion of 7 cases. Oral Surg. Oral Med. Oral Pathol. Oral Radiol. Endod. 94, 566-571 (2002).

127. Shipster, C. Oliver, B. \& Morgan, A. Speech and ora motor skills in children with Beckwith Wiedemann Syndrome: pre and post-tongue reduction surgery. Adv. Speech Lang. Pathol. 8, 45-55 (2006).

128. Shipster, C., Morgan, A. \& Dunaway, D. Psychosocial, feeding, and drooling outcomes in children with Beckwith Wiedemann syndrome following tongue reduction surgery. Cleft Palate. Craniofac. J. 49 e25-e34 (2012)

129. Heggie, a. a C., Vujcich, N. J. \& Portnof, J. E. and Morgan, a. T. Tongue reduction for macroglossia in Beckwith Wiedemann syndrome: review and application of new technique. Int. J. Oral Maxillofac. Surg. 42, 185-191 (2013).

130. Kamata, S. et al. Assessment of obstructive apnea by using polysomnography and surgical treatment in patients with Beckwith-Wiedemann syndrome. J. Pediatr. Surg. 40, E17-E19 (2005)

131. Cielo, C. M., Montalva, F. M. \& Taylor, J. A Craniofacial disorders associated with airway obstruction in the neonate. Semin. Fetal Neonatal Med. 21, 254-262 (2016).

132. Porter, A., Benson, C. B., Hawley, P. \& Wilkins-Haug, L. Outcome of fetuses with a prenatal ultrasound diagnosis of isolated omphalocele. Prenat. Diagn. 29 668-673 (2009)

133. Nessa, A., Rahman, S. A. \& Hussain, K. Hyperinsulinemic hypoglycemia - the molecular mechanisms. Front. Endocrinol. 7, 29 (2016)

134. Thornton, P. S. et al. Recommendations from the Pediatric Endocrine Society for Evaluation and Management of Persistent Hypoglycemia in Neonates, Infants, and Children. J. Pediatr. 167, 238-245 (2015).

135. Roženková, K., Güemes, M., Shah, P. \& Hussain, K. The diagnosis and management of hyperinsulinaemic hypoglycaemia. J. Clin. Res. Pediatr. Endocrinol. 7 86-97 (2015)

136. Güemes, M. et al. Severe hyperinsulinaemic hypoglycaemia in Beckwith-Wiedemann syndrome due to paternal uniparental disomy of $11 \mathrm{p} 15.5$ managed with sirolimus therapy. Horm. Res. Paediatr. 85, 353-357 (2016).

137. Kalish, J. M. et al. Clinical features of three girls with mosaic genome-wide paternal uniparental isodisomy. Am. J. Med. Genet. A 161 A, 1929-1939 (2013).

138. Astuti, D. et al. Germline mutations in DIS3L2 cause the Perlman syndrome of overgrowth and Wilms tumor susceptibility. Nat. Genet. 44, 277-284 (2012).

139. Kerr, B. et al. Genotype-phenotype correlation in Costello syndrome: HRAS mutation analysis in 43 cases. J. Med. Genet. 43, 401-405 (2006). 
140. Tatton-Brown, K. et al. Genotype-phenotype associations in Sotos syndrome: an analysis of 266 individuals with NSD 1 aberrations. Am. J. Hum. Genet. 77, 193-204 (2005)

141. Tatton-Brown, K. et al. Weaver syndrome and EZH2 mutations: Clarifying the clinical phenotype. Am. J. Med. Genet. A 161 A, 2972-2980 (2013).

142. Malan, V. et al. Distinct effects of allelic NFIX mutations on nonsense-mediated mRNA decay engender either a Sotos-like or a Marshall-Smith syndrome. Am. J. Hum. Genet. 87, 189-198 (2010).

143. Marsh, D. J. et al. Germline mutations in PTEN are present in Bannayan-Zonana syndrome. Nat. Genet 16, 333-334 (1997)

144. Lindhurst, M. J. et al. Mosaic overgrowth with fibroadipose hyperplasia is caused by somatic activating mutations in PIK3CA. Nat. Genet. 44, 928-933 (2012)

145. Gardiner, K. et al. Brain abnormalities in patients with Beckwith-Wiedemann syndrome. Am. J. Med. Genet. Part A 158A, 1388-1394 (2012).

146. Goldman, M. et al. Renal abnormalities in Beckwith Wiedemann syndrome are associated with $11 \mathrm{p} 15.5$ uniparental disomy. J. Am. Soc. Nephrol. 13, 2077-2084 (2002).

147. Goldman, M., Shuman, C., Weksberg, R. \& Rosenblum, N. Hypercalciuria in beckwith-wiedemann syndrome. J. Pediatr. 142, 206-208 (2003).

148. Fillion, M.-L., Watt, C. L. \& Gupta, I. R. Vesicoureteric reflux and reflux nephropathy: from mouse models to childhood disease. Pediatr. Nephrol. 29, 757-766 (2014).

149. Mussa, A. et al. Cancer risk in Beckwith-Wiedemann syndrome: a systematic review and meta-analysis outlining a novel (epi)genotype specific histotype targeted screening protocol. J. Pediatr. 176, 142-149. e1 (2016).

150. Brzezinski, J. et al. Wilms tumour in BeckwithWiedemann syndrome and loss of methylation at imprinting centre 2: revisiting tumour surveillance guidelines. Eur. J. Hum. Genet. 25, 1031-1039 (2017)

151. Brioude, F. et al. Answer to Wilms tumour in Beckwith-Wiedemann Syndrome and loss of methylation at imprinting centre 2: revisiting tumour surveillance guidelines. Eur. J. Hum. Genet. (in press).

152. Bertoin, F. et al. Genome-wide paternal uniparental disomy as a cause of Beckwith-Wiedemann syndrome associated with recurrent virilizing adrenocortical tumors. Horm. Metab. Res. 47, 497-503 (2015)

153. Bliek, J., Maas, S., Alders, M., Merks, J. H. M. \& Mannens, M. Epigenotype, phenotype, and tumors in patients with isolated hemihyperplasia. J. Pediatr. 153, 95-100 (2008)

154. Scott, R. H. et al. Surveillance for Wilms tumour in at-risk children: pragmatic recommendations for best practice. Arch. Dis. Child. 91, 995-999 (2006)

155. Kalish, J. M. et al. Surveillance recommendations for children with overgrowth syndromes and predisposition to Wilms tumors and hepatoblastoma. Clin. Cancer Res. 23, e115-e122 (2017).

156. Craft, A. W. Growth rate of Wilms' tumour. Lancet 354, 1127 (1999)

157. Choyke, P. L., Siegel, M. J., Craft, A. W., Green, D. M. $\&$ DeBaun, M. R. Screening for Wilms tumor in children with Beckwith-Wiedemann syndrome or idiopathic hemihypertrophy. Med. Pediatr. Oncol. 32 , 196-200 (1999).

158. McNeil, D. E., Brown, M., Ching, A. \& DeBaun, M. R. Screening for Wilms tumor and hepatoblastoma in children with Beckwith-Wiedemann syndromes: a costeffective model. Med. Pediatr. Oncol. 37, 349-356 (2001).

159. Rao, A., Rothman, J. \& Nichols, K. E. Genetic testing and tumor surveillance for children with cancer predisposition syndromes. Curr. Opin. Pediatr. 20 1-7 (2008).

160. Scalabre, A. et al. Is nephron sparing surgery justified in Wilms tumor with Beckwith-Wiedemann syndrome or isolated hemihypertrophy? Pediatr. Blood Cancer 63, 1571-1577 (2016)

161. Porteus, M. H. et al. Characteristics and outcome of children with Beckwith-Wiedemann syndrome and Wilms' tumor: a report from the national Wilms tumo study group. J. Clin. Oncol. 18, 2026-2031 (2000).

162. Dome, J. \& Huff, V. Wilms Tumor predisposition. GeneReviews http://www.ncbi.nlm.nih.gov/books/ nbk1294/ (2016).
163. Trobaugh-Lotrario, A. D., Venkatramani, R. \& Feusner, J. H. Hepatoblastoma in children with Beckwith-Wiedemann syndrome: does it warrant different treatment? J. Pediatr. Hematol. Oncol. 36 369-373 (2014).

164. Spector, L. G. \& Birch, J. The epidemiology of hepatoblastoma. Pediatr. Blood Cancer 59, 776-779 (2012).

165. Clericuzio, C. L. et al. Serum alpha-fetoprotein screening for hepatoblastoma in children with Beckwith-Wiedemann syndrome or isolated hemihyperplasia. J. Pediatr. 143, 270-272 (2003).

166. Tan, T. Y. \& Amor, D. J. Tumour surveillance in Beckwith-Wiedemann syndrome and hemihyperplasia: a critical review of the evidence and suggested guidelines for local practice. J. Paediatr. Child Health 42, 486-490 (2006).

167. Blohm, M. E., Vesterling-Hörner, D., Calaminus, G. \& Göbel, U. Alpha 1-fetoprotein (AFP) reference values in infants up to 2 years of age. Pediatr. Hematol. Oncol. 15, 135-142 (1998).

168. Mussa, A. et al. $\alpha$-Fetoprotein assay on dried blood spot for hepatoblastoma screening in children with overgrowth-cancer predisposition syndromes. Pediatr. Res. 76, 544-548 (2014).

169. Everman, D. B. et al. Serum $\alpha$-fetoprotein levels in Beckwith-Wiedemann syndrome. J. Pediatr. 137, 123-127 (2000).

170. Kalish, J. M. \& Deardorff, M. A. Tumor screening in Beckwith-Wiedemann syndrome - to screen or not to screen? Am. J. Med. Genet. A 170A, 2261-2264 (2016).

171. Duffy, K. A., Deardorff, M. A. \& Kalish, J. M. The utility of alpha-fetoprotein screening in BeckwithWiedemann syndrome. Am. J. Med. Genet. A 173A 581-584 (2017).

172. Mussa, A. \& Ferrero, G. B. Screening hepatoblastoma in Beckwith-Wiedemann syndrome: a complex issue. J. Pediatr. Hematol. Oncol. 37, 627 (2015).

173. Mussa, A. \& Ferrero, G. B. Serum alpha-fetoprotein screening for hepatoblastoma in Beckwith-Wiedemann syndrome. Am. J. Med. Genet. A 173A, 585-587 (2017).

174. Bessho, F., Hashizume, K., Nakajo, T. \& Kamoshita, S Mass screening in Japan increased the detection of infants with neuroblastoma without a decrease in cases in older children. J. Pediatr. 119, 237-241 (1991).

175. Yamamoto, K. et al. Mass screening and age-specific incidence of neuroblastoma in Saitama Prefecture. Japan. J. Clin. Oncol. 13, 2033-2038 (1995).

176. Ribeiro, R. C. et al. Adrenocortical tumors in children. Braz. J. Med. Biol. Res. 33, 1225-1234 (2000).

177. Bergeron, C. et al. Does nephroblastomatosis influence the natural history and relapse rate in Wilms tumour? A single centre experience over 11 years. Eur. J. Cancer 37, 385-391 (2001)

178. Clouston, W. M. et al. Virilizing adrenal adenoma in an adult with the Beckwith-Wiedemann syndrome: paradoxical response to dexamethasone. Clin. Endocrinol. 31, 467-473 (1989).

179. Brioude, F. et al. Hypercortisolism due to a pituitary adenoma associated with Beckwith-Wiedemann syndrome. Horm. Res. Paediatr. 86, 206-211 (2016).

180. Bémurat, L. et al. Successful laparoscopic operation of bilateral pheochromocytoma in a patient with Beckwith-Wiedemann syndrome. J. Hum. Hypertens. 16, 281-284 (2002)

181. Greer, K. J., Kirkpatrick, S. J., Weksberg, R. \& Pauli, R. M. Beckwith-Wiedemann syndrome in adults: observations from one family and recommendations for care. Am. J. Med. Genet. Part A 146, 1707-1712 (2008).

182. Moutou, C., Junien, C., Henry, I. \& Bonaïti-Pelliê, C. Beckwith-Wiedemann syndrome: a demonstration of the mechanisms responsible for the excess of transmitting females. J. Med. Genet. 29, 217-220 (1992)

183. Kent, L., Bowdin, S., Kirby, G. a, Cooper, W. N. \& Maher, E. R. Beckwith Weidemann syndrome: a behavioral phenotype-genotype study. Am. J. Med. Genet. B. Neuropsychiatr. Genet. 147B, 1295-1297 (2008)

184. Azzi, S. et al. Allele-specific methylated multiplex realtime quantitative PCR (ASMM RTQ-PCR), a powerful method for diagnosing loss of imprinting of the $11 \mathrm{p} 15$ region in Russell Silver and Beckwith Wiedemann syndromes. Hum. Mutat. 32, 249-258 (2011).

\section{Acknowledgements}

This Consensus Statement was organized by the European Network of Human Congenital Imprinting Disorders (EUCID. net) with financial support from European Cooperation in Science and Technology (COST; BM 1208). Newlife the Charity for Disabled Children, the European Society of Pediatric Endocrinology (ESPE) and the Société Française de lutte contre les Cancers et leucémies de l'enfant et de l'adolescent (SFCE) provided funding for the consensus meeting. The European Society of Pediatric Nephrology (ESPN) provided support for the meeting. Individual authors would like to thank the following funders for research support: Alex's Lemonade Stand Foundation (J.M.K.); Bundesministerium für Bildung und Forschung (BMBF) (number 01GM1513C) (D.P.); Child Growth Foundation (K.T.-B.); European Union FP7 Innovative Training Network (ITN) Ingenium N. 290123 (Y.LeB., A.R., I.N., E.R.M.); FIS (grant PI15/01481) (P.L., J.T.); Fondation de Recherche Médicale (Y.LeB.); Margaret Q. Landenberger Foundation (J.M.K.); MIUR PRIN 2015 JHLY35 (A.R., G.B.F., S.Ru.); MOH Grants to Istituto Auxologico Italiano (grant: RC 08C502 2015) (S.Ru.); US National Institutes of Health (grant K08CA193915) (J.M.K.); UK National Institute for Health Research (NIHR) Rare Diseases Translational Research Collaboration (A.C.F.); St. Baldrick's Scholar Award (J.M.K.); The Estonian Research Council (grant PUT355) (K.O.); Université P et M Curie, Institut National de la Santé Et de la Recherche Médicale (Y.L.B.); Telethon-Italia GGP15131 and AIRC IG 18671 (A.R.); Wellcome Trust (M.D.K.); European Research Council (E.R.M.); and NIHR Senior Investigator Award (E.R.M.). The University of Cambridge has received salary support in respect of E.R.M. from the National Health Service (NHS) in the East of England through the Clinical Academic Reserve. The views expressed are those of the authors and not necessarily those of the NHS or UK Department of Health. No funding was received from pharmaceutical companies. The authors thank L. Autelitano, C. Cielo, M. Deardorff, D. De León-Crutchlow, K. Duffy, A. Ganguly, D. Hobin, M. Meazzini, K. Pritchard-Jones, J. Taylor and R. Weksberg for their helpful input into the consensus statement. We apologize to the many authors whose work we were unable to cite because of space limitations.

\section{Author contributions}

F.B., J.M.K., A.M., A.C.F. J.B., S.E.B., A.I., D.P., Z.T., T.E D.J.G.M., A.R. and E.R.M. researched data for the article F.B., J.M.K., A.M., A.C.F., J.B., G.B.F., S.E.B., T.C., R.B., M.B., G.C., C.C., M.D.P., K.H., M.K.-W., C.P.K., Y.LeB., S.M.M., L.P., S.Ro., S.Ru., C.S., K.T.-B., J.T., C.T., K.G., I.N., R.C.H., D.P. Z.T. T.E. D.J.G.M., A.R., E.L., K.O., A.S and E.R.M. made substantial contribution to discussion of the content. F.B. J.M.K., A.M., A.C.F., J.B., S.E.B., A.I., M.D.K., F.M., C.S. R.C.H., D.P., Z.T., T.E., D.J.G.M., A.R. and E.R.M. wrote the article. All authors reviewed and/or edited the manuscript before submission.

\section{Competing interests statement}

The authors declare no competing interests.

\section{Publisher's note}

Springer Nature remains neutral with regard to jurisdictional claims in published maps and institutional affiliations.

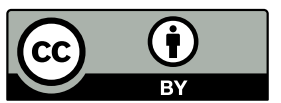

This work is licensed under a Creative Commons Attribution 4.0 International License. The images or other third party material in this article are included in the article's Creative Commons license unless indicated otherwise in the credit line; if the material is not included under the Creative Commons license, users will need to obtain permission from the license holder to reproduce the material. To view a copy of this license, visit http://creativecommons.org/licenses/by/4.0/.

SUPPLEMENTARY INFORMATION

See online article: $\underline{\mathrm{S} 1}$ (Table) $\underline{\underline{S} 2}$ (Figure) $\mid \underline{\mathrm{S}} \underline{3}$ (Table) $\mid$ $\underline{\mathrm{S}}$ (table) $\mid \underline{\mathrm{S}}$ (table)

FURTHER INFORMATION

European Network for Human Congenital Imprinting

Disorders: www.imprinting-disorders.eu

ALL LINKS ARE ACTIVE IN THE ONLINE PDF 